TRANSACTIONS OF THE

AMERICAN MATHEMATICAL SOCIETY

Volume 359, Number 9, September 2007, Pages 4183-4208

S 0002-9947(07)04378-4

Article electronically published on April 6, 2007

\title{
THE CAUCHY PROBLEM FOR IMPROPER AFFINE SPHERES AND THE HESSIAN ONE EQUATION
}

\author{
JUAN A. ALEDO, ROSA M. B. CHAVES, AND JOSÉ A. GÁLVEZ
}

\begin{abstract}
We give a conformal representation for improper affine spheres which is used to solve the Cauchy problem for the Hessian one equation. With this representation, we characterize the geodesics of an improper affine sphere, study its symmetries and classify the helicoidal ones. Finally, we obtain the complete classification of the isolated singularities of the Hessian one MongeAmpère equation.
\end{abstract}

\section{INTRODUCTION}

It is well known that the elliptic Monge-Ampère equations are one of the main research streams in partial differential equations. In particular, the classical MongeAmpère equation

$$
\operatorname{det}\left(\frac{\partial^{2} u}{\partial x^{i} \partial x^{j}}\right)=1
$$

is an important inquiry topic which has generated a large quantity of work. Its interest is also clear in differential geometry. Among other applications, the graphs of these solutions can be considered as improper affine spheres in affine differential geometry (see $[\mathrm{CY}]$ ), and they are also the integrability conditions for flat surfaces in hyperbolic 3-space (see, for instance, GMM] or [Sp, p. 165]).

The affine spheres in the unimodular affine 3-space are the totally umbilical surfaces of the affine theory, which studies the invariant properties under the group of unimodular affine transformations. They are the basic surfaces in this theory, with constant affine mean curvature $H$ and affine normal lines that are mutually parallel for improper affine spheres, i.e. $H=0$, or pass through a fixed point for proper affine spheres, i.e. $H \neq 0$. In contrast to the Euclidean case, the class of totally umbilical surfaces is very large, and it has been extensively investigated.

For the geometric study of the improper affine spheres we will consider its associated Cauchy problem: Find all improper affine spheres containing a prescribed curve and with a given analytic tangent plane distribution along the curve.

This problem can be considered as a generalization of the Cauchy problem for the above-mentioned Hessian one equation and it is inspired by the classical

Received by the editors June 29, 2005.

2000 Mathematics Subject Classification. Primary 53A15, 35J60; Secondary 53C45.

The first and third authors were partially supported by Ministerio de Education y Ciencia Grant No. MTM2004-02746. This work was started while the last author was visiting the IME at the University of Sao Paulo. He would like to thank all members of this institution for their hospitality.

(C)2007 American Mathematical Society Reverts to public domain 28 years from publication 
Björling problem for minimal surfaces in Euclidean 3-space, which was posed by E.G. Björling in 1844 and solved in 1890 by H.A. Schwarz [DHKW, pp. 120-135].

The importance of the Björling problem relies on the fact that it has been used to prove interesting geometric properties of minimal surfaces in $\mathbb{R}^{3}$. This has motivated the generalization of the Björling problem to other geometric theories, as well as its application to the local and global description of different families of surfaces. Among the references dealing with the classical Björling problem and its generalizations we may quote [ACM], Bl1], DHKW], GMi1, GMi2, GMi3, Mi], [Ni.

In affine differential geometry, a Björling type problem was first considered by W. Blaschke Bl1 for the class of affine surfaces with vanishing affine mean curvature, i.e., surfaces with maximal affinely invariant area Ca. Nevertheless, his study is centered in finding all maximal affine surfaces whose affine conormal contains a given analytic strip. Thus, the prescribed initial data are related to the affine conormal of the surface and not to the surface itself, which makes it difficult to give geometric properties for the maximal affine surface. The Blaschke solution to this problem depends strongly on the vanishing of the Laplacian of the affine conormal immersion, as happens in Björling's case for the coordinates of the minimal immersion. In contrast, we will fix initial data on the surface in order for it to be studied directly and will relate the problem to the above Monge-Ampère equation.

Thus, after some preliminaries, in Section 3 we study necessary and sufficient conditions on an analytic strip to contain an improper affine sphere and give a conformal representation which is adapted to solve the associated Cauchy problem. It is important to remark that our Weierstrass type resolution is different from the classical conformal representation for maximal affine surfaces by Blaschke [B12] and the one for improper affine spheres by L. Ferrer, A. Martínez and F. Milán [FMM1, FMM2, and it is particularly useful in the study of special curves on an improper affine sphere.

As an immediate consequence, we obtain conditions on an analytic curve $\beta$ for the existence of a unique improper affine sphere containing it when the affine metric is prescribed along $\beta$. In addition, using our conformal representation, we solve explicitly the Cauchy problem for the Hessian one Monge-Ampère equation.

In Section 4 we study the geodesics of an improper affine sphere. We characterize the geodesics for the affine metric and give a necessary and sufficient condition on a curve to be the geodesic of some improper affine sphere, which can be calculated using the resolution to the Cauchy problem. Moreover, we derive a reflection principle as well as a generalized symmetry principle and show how spheres with the topology of a cylinder can be computed.

Section 5 is devoted to the classification of the helicoidal improper affine spheres, that is, those which are invariant under a 1-parameter equiaffine isometry group. For this, we observe that the orbit of a fixed point is a curve with some symmetry properties, which allows us to use the resolution to the Cauchy problem. In addition, we investigate its affine completeness, when an orbit is considered as its boundary.

In Section 6, we focus our attention on the singularities of the Hessian one MongeAmpère equation. Two famous results by K. Jörgens [Jo1, [Jo2 showed that the surfaces of revolution provide the only entire solutions or entire solutions with an isolated singularity to this equation, up to equiaffine transformations. Recently, the 
classification of the entire solutions to this equation with a finite number of isolated singularities was achieved GMMi. However, nothing was known about the local classification of these singularities.

We prove that there exists an explicit one-to-one correspondence between the class of local solutions to the Monge-Ampère equation with an isolated singularity and the family of planar convex regular analytic Jordan curves. For this, we need to solve a slightly more general Cauchy problem for a class of Lagrangian immersions associated to the Hessian one equation [Wo and show how a curve of singularities of an improper affine sphere can determine the surface itself.

\section{Preliminaries}

In this section we examine some elementary facts concerning improper affine spheres and establish some notation. We refer the reader to [CY], [LSZ] and [NS] for more detailed discussions of affine surfaces.

Let $\left(x^{1}, x^{2}, x^{3}\right)$ be a rectangular coordinate system in $\mathbb{R}^{3}$ and $\psi: M \longrightarrow \mathbb{R}^{3}$ a (locally strongly convex) improper affine sphere with a possibly empty differentiable boundary. Then, up to an equiaffine transformation, we can assume that its affine normal is given by $e^{3}=(0,0,1)$.

Since the affine normal is transverse to the immersion, $\psi$ can be locally seen as the graph of an analytic strongly convex function $f\left(x^{1}, x^{2}\right)$ satisfying the Hessian one equation

$$
f_{x^{1} x^{1}} f_{x^{2} x^{2}}-f_{x^{1} x^{2}}^{2}=1
$$

with $f_{x^{i} x^{i}}>0$ for $i=1,2$. Here $f_{x^{i} x^{j}}$ stands for $\frac{\partial^{2} f}{\partial x^{i} \partial x^{j}}$.

In such a case, the affine conormal $N$ and the affine metric $h$ of $\psi$ are given by

$$
\begin{aligned}
& N=\left(-f_{x^{1}},-f_{x^{2}}, 1\right), \\
& h=f_{x^{1} x^{1}}\left(d x^{1}\right)^{2}+2 f_{x^{1} x^{2}} d x^{1} d x^{2}+f_{x^{2} x^{2}}\left(d x^{2}\right)^{2} .
\end{aligned}
$$

Now, if we consider $M$ as a Riemann surface with the structure induced by the affine metric, then taking $z=x^{1}+i f_{x^{2}}$ and $\zeta=f_{x^{1}}+i x^{2}$ one gets from (2.1)

$$
h=\frac{1}{f_{x^{2} x^{2}}}|d z|^{2}=\frac{1}{f_{x^{1} x^{1}}}|d \zeta|^{2},
$$

that is, $z$ and $\zeta$ are well-oriented conformal parameter immersions.

Finally, we observe that the graph of any solution to the Monge-Ampère equation (2.1) is an improper affine sphere with affine normal $e^{3}$ if $f_{x^{i} x^{i}}>0$ for $i=1,2$, or affine normal $-e^{3}$ otherwise. Thus, some properties of the solutions to this equation can be deduced from the improper affine spheres and vice versa.

\section{Conformal representation for improper affine spheres}

In this section we give a representation for improper affine spheres in terms of holomorphic data. The representation is adapted to the resolution of the associated Cauchy problem, which can be formulated as follows:

Let $I$ be an interval, $\beta: I \longrightarrow \mathbb{R}^{3}$ a regular analytic curve and $\Pi$ an analytic distribution of planes along $\beta$ such that $\beta^{\prime}(s) \in \Pi(s)$ for all $s \in I$. Given a nonzero vector $\xi$, find every improper affine sphere containing $\beta(I)$ with tangent plane $\Pi(s)$ at $\beta(s)$ for all $s \in I$ and affine normal vector $\xi$. 
If we denote by $V(s)$ the affine conormal at $\beta(s)$, we observe that the knowledge of $\Pi(s)$ and $\xi$ determine $V(s)$ since the affine conormal is orthogonal to $\Pi(s)$ and its inner product with $\xi$ is one. Obviously, the regular analytic curve $V$ determines $\Pi$.

Thus, given two regular analytic curves $\beta, V: I \longrightarrow \mathbb{R}^{3}$ we study necessary and sufficient conditions on these curves for the existence of an improper affine sphere containing $\beta(I)$ with affine conormal map $V(s)$ at $\beta(s)$.

Now, let $\psi: M \longrightarrow \mathbb{R}^{3}$ be an improper affine sphere with affine normal $\xi$ and affine conormal map $N: M \longrightarrow \mathbb{R}^{3}$. If we consider a regular analytic curve $\alpha: I \longrightarrow M$ and $\beta=\psi \circ \alpha, V=N \circ \alpha$, then $\left\langle\beta^{\prime}(s), V(s)\right\rangle=0$ because $V(s)$ is normal to the immersion; $\left\langle\beta^{\prime \prime}(s), V(s)\right\rangle=h\left(\beta^{\prime}(s), \beta^{\prime}(s)\right)>0$ since the affine metric is Riemannian, and $\langle V(s), \xi\rangle=1$.

Motivated by these facts, we say that a pair of regular analytic curves $\beta, V$ : $I \longrightarrow \mathbb{R}^{3}$ is admissible for a nonzero vector $\xi$ if

$$
\left\{\begin{array}{l}
\left\langle\beta^{\prime}(s), V(s)\right\rangle=0 \\
\left\langle\beta^{\prime \prime}(s), V(s)\right\rangle>0 \\
\langle\xi, V(s)\rangle=1
\end{array}\right.
$$

for all $s \in I$.

Thereby, if the Cauchy problem has a solution, the pair of curves $\beta, V$ must be admissible. Conversely, we prove that this condition is sufficient to find a unique solution to the Cauchy problem. More concretely,

Theorem 3.1. Let $I$ be an interval and $\beta, V: I \longrightarrow \mathbb{R}^{3}$ an admissible pair of curves for a nonzero vector $\xi$. Then there exists a unique improper affine sphere containing $\beta(I)$ with affine conormal $V(s)$ at $\beta(s)$ for all $s \in I$ and affine normal vector $\xi$.

Proof. Let us assume $\xi=e^{3}$ and take an admissible pair $\beta, V: I \longrightarrow \mathbb{R}^{3}$ for $\xi$ with $\beta(s)=\left(\beta^{1}(s), \beta^{2}(s), \beta^{3}(s)\right)$ and $V(s)=\left(V^{1}(s), V^{2}(s), 1\right)$. Let us suppose that there exists an improper affine sphere $\psi=\left(\psi^{1}, \psi^{2}, \psi^{3}\right)$ with affine conormal $N=\left(N^{1}, N^{2}, 1\right)$ containing $\beta(I)$ such that its affine conormal along $\beta$ is $V$. By the inverse function theorem there exists a local conformal parameter $z$ for the affine metric $h$, defined in a complex domain containing $I$, such that its real part is $s$, i.e. $z=s+i t$.

As was proven in the above section, $\psi^{1}-i N^{2}$ is a well-oriented conformal parameter for $h$. Thus, $\left(\psi^{1}-i N^{2}\right)(z)$ is a holomorphic function such that $\left(\psi^{1}-i N^{2}\right)(s)=$ $\beta^{1}(s)-i V^{2}(s)$. Therefore, by analyticity, we have that $\left(\psi^{1}-i N^{2}\right)(z)=\beta^{1}(z)-$ $i V^{2}(z)$, where $\beta^{1}(z), V^{2}(z)$ denote the holomorphic extensions of $\beta^{1}, V^{2}$, respectively. This equality holds on a certain domain where the holomorphic extensions of $\beta^{1}$ and $V^{2}$ exist. Analogously, we obtain $\left(-N^{1}+i \psi^{2}\right)(z)=-V^{1}(z)+i \beta^{2}(z)$.

Thus, one has

$$
\begin{array}{ll}
\psi^{1}(z)=\Re\left(\beta^{1}(z)-i V^{2}(z)\right), & N^{1}(z)=-\Re\left(-V^{1}(z)+i \beta^{2}(z)\right), \\
\psi^{2}(z)=\Im\left(-V^{1}(z)+i \beta^{2}(z)\right), & N^{2}(z)=-\Im\left(\beta^{1}(z)-i V^{2}(z)\right),
\end{array}
$$

where $\Re$ and $\Im$ stand for the real and imaginary parts of a complex number, respectively.

Moreover, since $\left\langle\psi_{z}, N\right\rangle=0$, it follows that $\psi^{3}$ can be calculated as

$$
\psi^{3}(z)=\psi^{3}\left(s_{0}\right)-\int_{s_{0}}^{z}\left(\psi_{w}^{1} N^{1}+\psi_{w}^{2} N^{2}\right) d w+\left(\psi_{\bar{w}}^{1} N^{1}+\psi_{\bar{w}}^{2} N^{2}\right) d \bar{w},
$$


with $s_{0} \in I$. Using that $\psi^{3}\left(s_{0}\right)=\beta\left(s_{0}\right)$, the immersion $\psi$ can be computed from (3.2) and (3.3) in terms of $\beta(s)$ and $V(s)$.

This fact proves the uniqueness part of the theorem for $\xi=e^{3}$, because the above expressions only depend on $\beta, V$ and the parameter $s$, but an analytic change of $s$ is nothing but a conformal change of parameter for the affine metric.

To prove existence, given an admissible pair $\beta, V: I \longrightarrow \mathbb{R}^{3}$ for $e^{3}$ we define the functions $\psi^{1}, \psi^{2}, N^{1}$ and $N^{2}$ as in (3.2). These functions can be well defined in a simply connected complex domain containing $I$ where the holomorphic extensions of $\beta$ and $V$ are possible, and since

$$
\frac{\partial}{\partial z}\left(\psi_{\bar{z}}^{1} N^{1}+\psi_{\bar{z}}^{2} N^{2}\right)=\frac{\partial}{\partial \bar{z}}\left(\psi_{z}^{1} N^{1}+\psi_{z}^{2} N^{2}\right)
$$

we can also define the real function

$$
\psi^{3}(z)=\beta^{3}\left(s_{0}\right)-\int_{s_{0}}^{z}\left(\psi_{w}^{1} N^{1}+\psi_{w}^{2} N^{2}\right) d w+\left(\psi_{\bar{w}}^{1} N^{1}+\psi_{\bar{w}}^{2} N^{2}\right) d \bar{w}
$$

for a fixed point $s_{0} \in I$.

It is clear that $\psi^{j}(s)=\beta^{j}(s)$ and $N^{j}(s)=V^{j}(s)$ for all $s \in I, j=1,2$. Thus, from (3.4),

$$
\psi^{3}(s)=\beta^{3}\left(s_{0}\right)-\int_{s_{0}}^{s}\left(\beta^{1^{\prime}}(r) V^{1}(r)+{\beta^{2}}^{\prime}(r) V^{2}(r)\right) d r=\beta^{3}(s)
$$

for all $s \in I$, since the pair $\beta, V$ is admissible for $e^{3}$.

Now, we prove that $\psi=\left(\psi^{1}, \psi^{2}, \psi^{3}\right)$ is an improper affine sphere in a complex domain containing $I$ with affine conormal $N=\left(N^{1}, N^{2}, 1\right)$ and affine normal $e^{3}$.

First, we observe that

$$
\psi_{z} \wedge \psi_{\bar{z}}=\left(\psi_{z}^{1} \psi_{\bar{z}}^{2}-\psi_{\bar{z}}^{1} \psi_{z}^{2}\right) N=2 i \Im\left(\psi_{z}^{1} \psi_{\bar{z}}^{2}\right) N
$$

where

$$
\Im\left(\psi_{z}^{1} \psi_{\bar{z}}^{2}\right)(s)=-\frac{1}{4}\left\langle\beta^{\prime \prime}(s), V(s)\right\rangle<0,
$$

and where by $\wedge$ we denote the cross product in $\mathbb{R}^{3}$. This implies that $\psi$ is an immersion in a complex neighbourhood of $I$ with normal direction given by $N$.

Since the third coordinate immersion of $N$ is nonzero, $\psi$ can be locally considered as a graph with respect to the plane $\Pi_{0}=\left\{\left(x^{1}, x^{2}, x^{3}\right) \in \mathbb{R}^{3}: x^{3}=0\right\}$. Thus, if we take the local parameters $(x, y)=\left(\psi^{1}, \psi^{2}\right)$, then a straightforward computation shows that

$$
\psi_{x}^{3}=-N^{1}, \quad \psi_{y}^{3}=-N^{2} \quad \text { and } \quad \psi_{x x}^{3} \psi_{y y}^{3}-\left(\psi_{x y}^{3}\right)^{2}=1
$$

with $\psi_{x x}^{3}, \psi_{y y}^{3}>0$. Therefore, $\psi$ is an improper affine sphere with affine conormal $N$ and affine normal $e^{3}$.

This completes the proof for $\xi=e^{3}$. The general case for an arbitrary $\xi$ can be easily deduced from the above one.

From now on, without loss of generality, we will assume that, up to an equiaffine transformation, the affine normal is $e^{3}$. Thus, from the above theorem we have the following conformal representation.

Theorem 3.2. Let $I$ be an interval and $\beta, V: I \longrightarrow \mathbb{R}^{3}$ an admissible pair of curves for $e^{3}$. Then, the unique improper affine sphere $\psi=\left(\psi^{1}, \psi^{2}, \psi^{3}\right)$ containing 
$\beta(I)$ with affine conormal $V(s)$ at $\beta(s)$ and affine normal $e^{3}$ can be calculated in a neighbourhood of $I$ in $\mathbb{C}$ as follows:

$$
\begin{aligned}
& N^{1}(z)=-\Re\left(-V^{1}(z)+i \beta^{2}(z)\right), \\
& N^{2}(z)=-\Im\left(\beta^{1}(z)-i V^{2}(z)\right), \\
& \psi^{1}(z)=\Re\left(\beta^{1}(z)-i V^{2}(z)\right), \\
& \psi^{2}(z)=\Im\left(-V^{1}(z)+i \beta^{2}(z)\right), \\
& \psi^{3}(z)=\beta^{3}\left(s_{0}\right)-\int_{s_{0}}^{z}\left(\psi_{w}^{1} N^{1}+\psi_{w}^{2} N^{2}\right) d w+\left(\psi_{\bar{w}}^{1} N^{1}+\psi_{\bar{w}}^{2} N^{2}\right) d \bar{w},
\end{aligned}
$$

where $s_{0} \in I$ is a fixed point and $N=\left(N^{1}, N^{2}, 1\right)$ is its affine conormal.

Moreover, its affine metric $h$ is given by

$$
h=-\Re\left(\left(\beta_{z}^{1}-i V_{z}^{2}\right)\left(\overline{V_{z}^{1}}+i \overline{\beta_{z}^{2}}\right)\right)|d z|^{2} .
$$

Proof. We only need to compute the affine metric of the improper affine sphere, that is,

$$
\begin{aligned}
& h\left(\psi_{z}, \psi_{z}\right)=\left\langle\psi_{z z}, N\right\rangle=-\left\langle\psi_{z}, N_{z}\right\rangle=0, \\
& h\left(\psi_{z}, \psi_{\bar{z}}\right)=\left\langle\psi_{z \bar{z}}, N\right\rangle=\psi_{z \bar{z}}^{3} .
\end{aligned}
$$

Thus, using the last equation in 3.5.

$$
\psi_{z \bar{z}}^{3}=-\frac{1}{2} \Re\left(\left(\beta_{z}^{1}-i V_{z}^{2}\right)\left(\overline{V_{z}^{1}}+i \overline{\beta_{z}^{2}}\right)\right)
$$

and the theorem follows.

Remark 3.1. Although the conformal resolution given in the above theorem is local, it is clear that the global solution can be found by analytic continuation.

It is interesting to observe that for an admissible pair of curves $\beta, V$, the value of $V$ is determined, in some cases, in terms of the affine metric along $\beta$. More explicitly,

Lemma 3.1. Let $I$ be an interval and $\beta, V$ an admissible pair of curves for $e^{3}$. Let us consider the analytic function $m(s)=\left\langle\beta^{\prime \prime}(s), V(s)\right\rangle, s \in I$. If there exists $s_{0} \in I$ such that $\operatorname{det}\left(\beta^{\prime}\left(s_{0}\right), \beta^{\prime \prime}\left(s_{0}\right), e^{3}\right) \neq 0$, then $V$ can be calculated in terms of $\beta$ and $m$.

Proof. Since $\operatorname{det}\left(\beta^{\prime}\left(s_{0}\right), \beta^{\prime \prime}\left(s_{0}\right), e^{3}\right) \neq 0$ we obtain that $\operatorname{det}\left(\beta^{\prime}(s), \beta^{\prime \prime}(s), e^{3}\right) \neq 0$ in a neighbourhood of $s_{0}$. Thus, using that the pair is admissible, the system of linear equations with variable $V(s)$,

$$
\left\{\begin{array}{l}
\left\langle\beta^{\prime}(s), V(s)\right\rangle=0, \\
\left\langle\beta^{\prime \prime}(s), V(s)\right\rangle=m(s), \\
\left\langle e^{3}, V(s)\right\rangle=1,
\end{array}\right.
$$

can be solved in the above neighbourhood of $s_{0}$, with a unique solution. Thereby, $V$ is determined around $s_{0}$ and globally determined by analyticity.

If we write $\beta=\left(\beta^{1}, \beta^{2}, \beta^{3}\right)$, we have $\operatorname{det}\left(\beta^{\prime}, \beta^{\prime \prime}, e^{3}\right)=\beta^{1^{\prime}} \beta^{2^{\prime \prime}}-\beta^{2^{\prime}} \beta^{1^{\prime \prime}}$. Then, $\beta^{1^{\prime}} \beta^{2^{\prime \prime}}-\beta^{2^{\prime}} \beta^{1^{\prime \prime}} \neq 0$ if and only if the planar curve $\left(\beta^{1}, \beta^{2}\right)$ has nonzero Euclidean curvature.

Therefore, the condition $\operatorname{det}\left(\beta^{\prime}\left(s_{0}\right), \beta^{\prime \prime}\left(s_{0}\right), e^{3}\right) \neq 0$ is equivalent to saying that the affine projection of $\beta$ in the direction of $e^{3}$ on a transversal plane (and, hence, on every transversal plane) is a locally strongly convex curve at $s_{0}$. 
As a converse of the lemma we have

Proposition 3.1. Let $I$ be an interval and $\beta: I \longrightarrow \mathbb{R}^{3}$ a regular analytic curve such that $\operatorname{det}\left(\beta^{\prime}(s), \beta^{\prime \prime}(s), e^{3}\right) \neq 0$ for all $s \in I$. Then, given a positive analytic function $m: I \longrightarrow \mathbb{R}^{+}$there exists a unique improper affine sphere containing $\beta(I)$ and satisfying $h\left(\beta^{\prime}(s), \beta^{\prime}(s)\right)=m(s), s \in I$, where $h$ denotes its affine metric.

Proof. Bearing in mind that $h\left(\beta^{\prime}(s), \beta^{\prime}(s)\right)=\left\langle\beta^{\prime \prime}(s), V(s)\right\rangle$, the proof of this result is an easy consequence of (3.6) and Theorem 3.1

Example 3.1. Let us consider $\beta: \mathbb{R} \longrightarrow \mathbb{R}^{3}$ given by $\beta(s)=\left(b_{0} \cos s, b_{0} \sin s, 0\right)$ with $b_{0}>0$ and $m(s)=m_{0}>0$. Then Proposition 3.1 assures the existence of a unique improper affine sphere $\psi=\left(\psi^{1}, \psi^{2}, \psi^{3}\right)$ containing the circle $\beta(\mathbb{R})$ with constant affine metric $m_{0}$ on $\beta(\mathbb{R})$. This example provides us every improper affine sphere of revolution (see Section 5), which can be calculated from (3.6) and Theorem 3.2. That is,

$$
V(s)=\left(-\frac{m_{0}}{b_{0}} \cos s,-\frac{m_{0}}{b_{0}} \sin s, 1\right)
$$

and

$$
\begin{aligned}
\psi^{1}(s, t) & =b_{0} \cos s \cosh t-\frac{m_{0}}{b_{0}} \cos s \sinh t \\
\psi^{2}(s, t) & =-\frac{m_{0}}{b_{0}} \sin s \sinh t+b_{0} \sin s \cosh t \\
\psi^{3}(s, t) & =-\frac{1}{4 b_{0}^{2}}\left(2\left(m_{0}^{2}-b_{0}^{4}\right) t-2 m_{0} b_{0}^{2} \cosh (2 t)+\left(m_{0}^{2}+b_{0}^{4}\right) \sinh (2 t)\right)-\frac{m_{0}}{2}
\end{aligned}
$$

where we have used the holomorphic extensions of $\cos s$ and $\sin s$, i.e.,

$$
\begin{aligned}
& \cos z=\cos s \cosh t-i \sin s \sinh t, \\
& \sin z=\sin s \cosh t+i \cos s \sinh t
\end{aligned}
$$

for $z=s+i t$.

Remark 3.2. Let us consider the regular analytic curve $\beta: \mathbb{R} \longrightarrow \mathbb{R}^{3}$ given by $\beta(s)=(\cos s, s, \sin s)$. In this case $\operatorname{det}\left(\beta^{\prime}(s), \beta^{\prime \prime}(s), e^{3}\right)=\cos s$ and, so, if we take $m(s)=1$ for all $s \in \mathbb{R}$, then from (3.6) we have

$$
\left.V(s)=\left(-\frac{1}{\cos s}-\tan s,-\cos s-(1+\sin s) \tan s, 1\right), \quad s \in\right]-\frac{\pi}{2}, \frac{\pi}{2}[.
$$

This proves that the condition $\operatorname{det}\left(\beta^{\prime}(s), \beta^{\prime \prime}(s), e^{3}\right) \neq 0$ at any point in Proposition 3.1 cannot be relaxed, since it is not possible to define $V$ in $\mathbb{R}$.

Now, our aim is to solve the Cauchy problem for the classical Monge-Ampère equation

$$
\left\{\begin{array}{l}
f_{x x} f_{y y}-f_{x y}^{2}=1, \\
f(x, 0)=a(x), \\
f_{y}(x, 0)=b(x),
\end{array} \quad a^{\prime \prime}(x)>0,\right.
$$

where $a, b$ are two analytic functions defined on an interval $I$ and $f$ is defined on a domain in the $(x, y)$-plane containing $I \times\{0\}$.

Note that $a^{\prime \prime}(x)$ must be different from zero in order for the Monge-Ampère equation to be satisfied. Thereby, without loss of generality, changing $f$ to $-f$ if necessary, we can assume $a^{\prime \prime}(x)>0$ for all $x \in I$. 
Bearing in mind that the graph of a solution of the Monge-Ampère equation can be locally seen as an improper affine sphere, one has

Theorem 3.3. There exists a unique solution to the Cauchy problem (3.7) in a neighbourhood of $I \times\{0\}$ given by

$$
\begin{aligned}
f(x, y)=a\left(x_{0}\right)+ & \frac{1}{2} \int_{x_{0}}^{z}\left\{\Re\left(a^{\prime}(w)\left(1+i b^{\prime}(w)\right)\right)-i \Im(w+i b(w)) a^{\prime \prime}(w)\right\} d w \\
& +\left\{\Re\left(a^{\prime}(w)\right)\left(1-i \overline{b^{\prime}(w)}\right)+i \Im(w+i b(w)) \overline{a^{\prime \prime}(w)}\right\} d \bar{w}
\end{aligned}
$$

with

$$
x(z)=\Re(z)-\Im(b(z)), \quad y(z)=\Im\left(a^{\prime}(z)\right) .
$$

Here, $a(z)$ and $b(z)$ are holomorphic extensions of $a(x)$ and $b(x)$, respectively, and $x_{0} \in I$ is a fixed point.

Proof. It is easy to convince oneself that solving the Cauchy problem (3.7) is equivalent to solving the Cauchy problem for improper affine spheres and admissible data $\beta(s)=(s, 0, a(s)), V(s)=\left(-a^{\prime}(s),-b(s), 1\right), s \in I$, for $\xi=e^{3}$ (see Section 2).

Then, the result follows from Theorem 3.1 and Theorem 3.2 .

\section{Some Applications}

It is well known that a curve of an improper affine sphere is a pre-geodesic for the induced connection if and only if the affine projection of the curve in the direction of the affine normal $\xi$ on a transversal plane lies in a line (see, for instance, [NS]). Here, we mean by pre-geodesic a geodesic up to reparametrizations.

We use the resolution to the Cauchy problem in order to characterize the geodesics of an improper affine sphere for the affine metric and to study what are the conditions that a given curve must satisfy to be the geodesic of some improper affine sphere.

Theorem 4.1. Let $M$ be a Riemann surface and $\psi: M \longrightarrow \mathbb{R}^{3}$ an improper affine sphere with affine conormal $N: M \longrightarrow \mathbb{R}^{3}$. If we consider a regular analytic curve $\alpha: I \longrightarrow M$ from an interval $I$ and take $\beta=\psi \circ \alpha, V=N \circ \alpha$, then $\beta$ is a pre-geodesic for the affine metric iff $\operatorname{det}\left(\beta^{\prime}(s), \beta^{\prime \prime}(s), e^{3}\right)+\operatorname{det}\left(V^{\prime}(s), V^{\prime \prime}(s), e^{3}\right)=0$ for all $s \in I$.

Proof. Using Theorem 3.2 we have a conformal parameter $z=s+i t$, defined in a neighbourhood containing $I$, for the affine metric $h$ such that $\psi=\beta$ for $z=s$.

If we denote by $\widehat{\nabla}$ the Levi-Civita connection of $h$, then $\beta$ is a pre-geodesic if and only if $\widehat{\nabla}_{\beta^{\prime}(s)} \beta^{\prime}(s)$ is proportional to $\beta^{\prime}(s)$. Or equivalently,

$$
0=h\left(\widehat{\nabla}_{\frac{\partial}{\partial s}} \frac{\partial}{\partial s}, \frac{\partial}{\partial t}\right)=-\frac{1}{2} \frac{\partial}{\partial t} h\left(\frac{\partial}{\partial s}, \frac{\partial}{\partial s}\right)=-\frac{\partial}{\partial t} h\left(\frac{\partial}{\partial z}, \frac{\partial}{\partial \bar{z}}\right)
$$

along $\beta(s)$, that is,

$$
\Im\left(\frac{\partial}{\partial z} h\left(\frac{\partial}{\partial z}, \frac{\partial}{\partial \bar{z}}\right)\right)=0
$$

for all $z=s \in I$.

Thus, deriving with respect to $z$ the expression of $h$ given in Theorem 3.2 and evaluating at $z=s$ one has that the imaginary part of

$$
\left(\beta^{1^{\prime \prime}}(s)-i V^{2^{\prime \prime}}(s)\right)\left(V^{1^{\prime}}(s)+i \beta^{2^{\prime}}(s)\right)+\left(\beta^{1^{\prime}}(s)+i V^{2^{\prime}}(s)\right)\left(V^{1^{\prime \prime}}(s)-i \beta^{2^{\prime \prime}}(s)\right)
$$


must vanish identically. That is,

$$
\begin{aligned}
0 & =\beta^{1^{\prime}}(s) \beta^{2^{\prime \prime}}(s)-\beta^{1^{\prime \prime}}(s) \beta^{2^{\prime}}(s)+V^{1^{\prime}}(s) V^{2^{\prime \prime}}(s)-V^{1^{\prime \prime}}(s) V^{2^{\prime}}(s) \\
& =\operatorname{det}\left(\beta^{\prime}(s), \beta^{\prime \prime}(s), e^{3}\right)+\operatorname{det}\left(V^{\prime}(s), V^{\prime \prime}(s), e^{3}\right),
\end{aligned}
$$

as we wanted to prove.

Bearing in mind that a pre-geodesic $\beta$ is a geodesic if and only if

$$
0=h\left(\widehat{\nabla}_{\beta^{\prime}(s)} \beta^{\prime}(s), \beta^{\prime}(s)\right)=\frac{\partial}{\partial s} h\left(\beta^{\prime}(s), \beta^{\prime}(s)\right),
$$

one has

Corollary 4.1. Let $I$ be an interval and $\beta: I \longrightarrow \mathbb{R}^{3}$ a regular analytic curve. Then $\beta$ is the geodesic of some improper affine sphere for the affine metric if and only if there exists a regular analytic curve $V: I \longrightarrow \mathbb{R}^{3}$ satisfying

$$
\left\{\begin{array}{l}
\left\langle\beta^{\prime}(s), V(s)\right\rangle=0 \\
\left\langle\beta^{\prime \prime}(s), V(s)\right\rangle=c_{0} \\
\left\langle e^{3}, V(s)\right\rangle=1
\end{array}\right.
$$

for a positive constant $c_{0}$, such that $\operatorname{det}\left(\beta^{\prime}(s), \beta^{\prime \prime}(s), e^{3}\right)+\operatorname{det}\left(V^{\prime}(s), V^{\prime \prime}(s), e^{3}\right)=0$.

Example 4.1. It is easy to check that the curve $\beta(s)=(\cos s, \sin s, 0)$ cannot be a geodesic of an improper affine sphere for the affine metric. The only curve $V$ verifying (4.1) is $V(s)=(-\cos s,-\sin s, 1)$. But,

$$
\operatorname{det}\left(\beta^{\prime}(s), \beta^{\prime \prime}(s), e^{3}\right)+\operatorname{det}\left(V^{\prime}(s), V^{\prime \prime}(s), e^{3}\right)=1+c_{0}^{2} .
$$

However, for the curve $\beta(s)=(\cosh s, \sinh s, 0)$ there exists a unique improper affine sphere having $\beta$ as a geodesic for the affine metric which can be calculated by taking $V(s)=(\cosh s,-\sinh s, 1)$.

Now, we use the conformal representation for improper affine spheres in order to establish several consequences regarding their symmetries. Our aim is to see how a symmetry in the admissible data generates a symmetry of the whole surface.

Let $F: \mathbb{R}^{3} \longrightarrow \mathbb{R}^{3}$ be an element of the group $A\left(\mathbb{R}^{3}\right)$, that is, an affine transformation with $\operatorname{det}(d F)= \pm 1$. As is well known, $F$ can be written as

$$
F(v)=A v+b, \quad v \in \mathbb{R}^{3},
$$

where $A$ is a $3 \times 3$ matrix with determinant \pm 1 and $b \in \mathbb{R}^{3}$ is a fixed vector. We observe that if $\psi$ is an improper affine sphere with affine conormal vector field $N$ and affine normal $\xi$, then $F \circ \psi$ can be seen as an improper affine sphere with affine conormal $\left(A^{t}\right)^{-1} N$ and affine normal $A \xi$.

Thus, given an admissible pair of curves $\beta, V: I \longrightarrow \mathbb{R}^{3}$ for $\xi=e^{3}$, we will say that $F \in A\left(\mathbb{R}^{3}\right)$ is a symmetry of the admissible pair if $A e^{3}=e^{3}$ and there exists an analytic diffeomorphism $f: I \longrightarrow I$ such that $\beta \circ f=F \circ \beta$ and $V \circ f=\left(A^{t}\right)^{-1} V$.

Theorem 4.2 (Generalized symmetry principle). Any symmetry of an admissible pair generates a global symmetry of the resulting improper affine sphere.

Corollary 4.2. Let $\beta, V: \mathbb{R} \longrightarrow \mathbb{R}^{3}$ be an admissible pair of T-periodic regular curves for some $T>0$. Then the improper affine sphere that they generate has the topology of a cylinder in a neighbourhood of $\beta(\mathbb{R})$.

Conversely, every improper affine sphere with the topology of a cylinder can be constructed in this way. 
The proofs of these results are, in essence, analogous to the corresponding ones in GMi2, and so we omit them.

A particular case occurs when a vertical plane intersects orthogonally an improper affine sphere:

Corollary 4.3 (Reflection principle). Every vertical plane which intersects orthogonally an improper affine sphere is a reflection plane of the surface.

Proof. Let us denote by $\beta$ the curve (locally) given by the intersection of the vertical plane $\Pi$ and the improper affine sphere, and by $V$ its affine conormal along $\beta$. If we consider the orthogonal reflection $F$ with respect to $\Pi$, it is clear that $d F\left(e^{3}\right)=e^{3}$ and $V$ lies on $\Pi$.

Thus, $F$ is a symmetry of $\beta, V$, with $f=I d$, and, from Theorem $4.2, F$ is also a symmetry of the surface.

The generalized symmetry principle provides us the main tool for the classification of the helicoidal improper affine spheres. Specifically, we will use the following result, which is very useful for the construction of improper affine spheres with prescribed symmetries.

Corollary 4.4. Let $\beta: I \longrightarrow \mathbb{R}^{3}$ be a regular curve satisfying $\operatorname{det}\left(\beta^{\prime}(s), \beta^{\prime \prime}(s), e^{3}\right) \neq$ $0, s \in I$, and $m: I \longrightarrow \mathbb{R}^{+}$an analytic function. If $F \in A\left(\mathbb{R}^{3}\right)$ and $f: I \longrightarrow I$ is an analytic diffeomorphism such that $d F\left(e^{3}\right)=e^{3}, F \circ \beta=\beta \circ f$ and $m(s)=$ $f^{\prime}(s)^{2} m(f(s))$, then $F$ is a symmetry of the improper affine sphere given by Proposition 3.1 .

Proof. Let $V(s)$ be the unique solution to (3.6) and let us write $F$ as in (4.2). Then, using that $\beta(f(s))=F(\beta(s))=A \beta(s)+b$, we obtain

$$
\beta^{\prime}(f(s))=\frac{1}{f^{\prime}(s)} A \beta^{\prime}(s), \quad \beta^{\prime \prime}(f(s))=\frac{1}{f^{\prime}(s)^{2}}\left(A \beta^{\prime \prime}(s)-f^{\prime \prime}(s) \beta^{\prime}(f(s))\right)
$$

and (3.6) becomes

$$
\left\{\begin{array}{l}
\left\langle A \beta^{\prime}(s), V(f(s))\right\rangle=0 \\
\left\langle A \beta^{\prime \prime}(s), V(f(s))\right\rangle=f^{\prime}(s)^{2} m(f(s))=m(s), \\
\left\langle A e^{3}, V(f(s))\right\rangle=1 .
\end{array}\right.
$$

Thus $V(f(s))=\left(A^{t}\right)^{-1} V(s)$ is the only solution to the above linear system and the result follows from the generalized symmetry principle.

Example 4.2. Let us consider the curve $\beta: \mathbb{R} \longrightarrow \mathbb{R}^{3}$ given by $\beta(s)=(\cos (s)$, $\sin (s), 0)$ and $m(s)=1+\cos ^{2}(s)$. Then the unique improper affine sphere containing the circle $\beta(\mathbb{R})$ and affine metric $h$ such that $h\left(\beta^{\prime}(s), \beta^{\prime}(s)\right)=m(s)$ has, from Corollary 4.2, the topology of a cylinder, since Proposition 3.1 assures that $V(s)$ must be $2 \pi$-periodic as $\beta(s)$ and $m(s)$.

Moreover, if we take $F$ as the reflection with respect to the vertical plane with equation $x^{1}=0$ and $f(s)=-s$, we observe that, from Corollary 4.4, $F$ is a symmetry of the surface. Similarly, $x^{2}=0$ is another plane of symmetry of the improper affine sphere. 


\section{HELICOIDAL IMPROPER AFFINE SPHERES}

If $\psi$ is an improper affine sphere which remains unchanged by an equiaffine transformation $F$, then it is clear that $d F$ preserves the affine normal of $\psi$. Thus, for the study of the helicoidal improper affine spheres, we consider the equiaffine transformations whose differential preserves a nonzero fixed vector. Specifically, we denote by $\mathcal{G}$ the group of equiaffine transformations $F: \mathbb{R}^{3} \longrightarrow \mathbb{R}^{3}$ satisfying $d F\left(e^{3}\right)=e^{3}$.

We will identify $\mathcal{G}$, in a standard way, with the subgroup of matrices of $\mathbb{S L}(4, \mathbb{R})$ given by

$$
\left\{\left(\begin{array}{llll}
a & b & 0 & c \\
d & e & 0 & f \\
g & h & 1 & j \\
0 & 0 & 0 & 1
\end{array}\right): a, b, c, d, e, f, g, h, j \in \mathbb{R}, \text { with } a e-b d=1\right\},
$$

with Lie algebra

$$
\mathfrak{g}=\left\{\left(\begin{array}{cccc}
a & b & 0 & f \\
c & -a & 0 & g \\
d & e & 0 & h \\
0 & 0 & 0 & 0
\end{array}\right): a, b, c, d, e, f, g, h \in \mathbb{R}\right\} .
$$

Thus, one has

Proposition 5.1. Up to conjugation in $A\left(\mathbb{R}^{3}\right)$, every nontrivial 1-parameter subgroup of $\mathcal{G}$ is given by

$$
\begin{gathered}
\left(\begin{array}{l}
x^{1} \\
x^{2} \\
x^{3}
\end{array}\right) \longrightarrow\left(\begin{array}{ccc}
1 & 0 & 0 \\
s & 1 & 0 \\
\frac{a s^{2}}{2} & a s & 1
\end{array}\right)\left(\begin{array}{l}
x^{1} \\
x^{2} \\
x^{3}
\end{array}\right)+b\left(\begin{array}{c}
s \\
\frac{s^{2}}{2} \\
\frac{a s^{3}}{6}
\end{array}\right) \\
\left(\begin{array}{l}
x^{1} \\
x^{2} \\
x^{3}
\end{array}\right) \longrightarrow\left(\begin{array}{lll}
1 & 0 & 0 \\
s & 1 & 0 \\
0 & 0 & 1
\end{array}\right)\left(\begin{array}{l}
x^{1} \\
x^{2} \\
x^{3}
\end{array}\right)+\left(\begin{array}{c}
0 \\
0 \\
a s
\end{array}\right) \\
\left(\begin{array}{l}
x^{1} \\
x^{2} \\
x^{3}
\end{array}\right) \longrightarrow\left(\begin{array}{ccc}
\cosh s & \sinh s & 0 \\
\sinh s & \cosh s & 0 \\
0 & 0 & 1
\end{array}\right)\left(\begin{array}{l}
x^{1} \\
x^{2} \\
x^{3}
\end{array}\right)+\left(\begin{array}{c}
0 \\
0 \\
a s
\end{array}\right) \\
\left(\begin{array}{l}
x^{1} \\
x^{2} \\
x^{3}
\end{array}\right) \longrightarrow\left(\begin{array}{ccc}
\cos s & -\sin s & 0 \\
\sin s & \cos s & 0 \\
0 & 0 & 1
\end{array}\right)\left(\begin{array}{l}
x^{1} \\
x^{2} \\
x^{3}
\end{array}\right)+\left(\begin{array}{c}
0 \\
0 \\
a s
\end{array}\right) \\
\left(\begin{array}{l}
x^{1} \\
x^{2} \\
x^{3}
\end{array}\right) \\
\left(\begin{array}{lll}
x^{1} \\
x^{2} \\
x^{3}
\end{array}\right)
\end{gathered}
$$




$$
\left(\begin{array}{l}
x^{1} \\
x^{2} \\
x^{3}
\end{array}\right) \longrightarrow\left(\begin{array}{lll}
1 & 0 & 0 \\
0 & 1 & 0 \\
0 & 0 & 1
\end{array}\right)\left(\begin{array}{l}
x^{1} \\
x^{2} \\
x^{3}
\end{array}\right)+\left(\begin{array}{l}
0 \\
0 \\
s
\end{array}\right)
$$

for some constants $a, b \in \mathbb{R}$.

Proof. Let us consider the matrices $A \in \mathfrak{g}$ and $D_{1} \in \mathcal{G}$ given by

$$
A=\left(\begin{array}{cccc}
a & b & 0 & f \\
c & -a & 0 & g \\
d & e & 0 & h \\
0 & 0 & 0 & 0
\end{array}\right), \quad D_{1}=\left(\begin{array}{cccc}
\alpha & \beta & 0 & 0 \\
\gamma & \delta & 0 & 0 \\
0 & 0 & 1 & 0 \\
0 & 0 & 0 & 1
\end{array}\right)
$$

Then, by choosing suitable numbers $\alpha, \beta, \gamma, \delta$, the matrix $A_{1}=D_{1} A D_{1}^{-1}$ can be reduced to one of the following:

$$
\left(\begin{array}{cccc}
0 & 0 & 0 & f_{1} \\
c_{1} & 0 & 0 & g_{1} \\
d_{1} & e_{1} & 0 & h_{1} \\
0 & 0 & 0 & 0
\end{array}\right),\left(\begin{array}{cccc}
0 & c_{1} & 0 & f_{1} \\
c_{1} & 0 & 0 & g_{1} \\
d_{1} & e_{1} & 0 & h_{1} \\
0 & 0 & 0 & 0
\end{array}\right),\left(\begin{array}{cccc}
0 & -c_{1} & 0 & f_{1} \\
c_{1} & 0 & 0 & g_{1} \\
d_{1} & e_{1} & 0 & h_{1} \\
0 & 0 & 0 & 0
\end{array}\right),\left(\begin{array}{cccc}
a_{1} & 0 & 0 & f_{1} \\
0 & -a_{1} & 0 & g_{1} \\
d_{1} & e_{1} & 0 & h_{1} \\
0 & 0 & 0 & 0
\end{array}\right)
$$

with $c_{1} \neq 0$.

Now, we take $D_{2} \in \mathcal{G}$ as

$$
\left(\begin{array}{llll}
1 & 0 & 0 & \alpha \\
0 & 1 & 0 & \beta \\
\gamma & \delta & 1 & 0 \\
0 & 0 & 0 & 1
\end{array}\right) \quad \text { or } \quad\left(\begin{array}{cccc}
0 & 1 & 0 & \alpha \\
-1 & 0 & 0 & \beta \\
\gamma & \delta & 1 & 0 \\
0 & 0 & 0 & 1
\end{array}\right)
$$

Then, $A_{2}=D_{2} A_{1} D_{2}^{-1}$ is simplified in each case for appropriate numbers $\alpha, \beta, \gamma, \delta$ to one of these matrices:

$$
\begin{aligned}
& \left(\begin{array}{cccc}
0 & 0 & 0 & f_{2} \\
c_{1} & 0 & 0 & 0 \\
0 & e_{2} & 0 & 0 \\
0 & 0 & 0 & 0
\end{array}\right),\left(\begin{array}{cccc}
0 & 0 & 0 & 0 \\
c_{1} & 0 & 0 & 0 \\
0 & 0 & 0 & h_{2} \\
0 & 0 & 0 & 0
\end{array}\right),\left(\begin{array}{cccc}
0 & c_{1} & 0 & 0 \\
c_{1} & 0 & 0 & 0 \\
0 & 0 & 0 & h_{2} \\
0 & 0 & 0 & 0
\end{array}\right),\left(\begin{array}{cccc}
0 & -c_{1} & 0 & 0 \\
c_{1} & 0 & 0 & 0 \\
0 & 0 & 0 & h_{2} \\
0 & 0 & 0 & 0
\end{array}\right), \\
& \left(\begin{array}{cccc}
a_{1} & 0 & 0 & 0 \\
0 & -a_{1} & 0 & 0 \\
0 & 0 & 0 & h_{2} \\
0 & 0 & 0 & 0
\end{array}\right),\left(\begin{array}{cccc}
0 & 0 & 0 & f_{3} \\
0 & 0 & 0 & 0 \\
d_{2} & e_{2} & 0 & 0 \\
0 & 0 & 0 & 0
\end{array}\right),\left(\begin{array}{cccc}
0 & 0 & 0 & 0 \\
0 & 0 & 0 & 0 \\
0 & e_{3} & 0 & 0 \\
0 & 0 & 0 & 0
\end{array}\right),\left(\begin{array}{cccc}
0 & 0 & 0 & 0 \\
0 & 0 & 0 & 0 \\
0 & 0 & 0 & h_{3} \\
0 & 0 & 0 & 0
\end{array}\right),
\end{aligned}
$$

where $c_{1}, a_{1}, f_{3}, e_{3}, h_{3}$ are nonzero real numbers.

Since every continuous 1-parameter subgroup of $\mathcal{G}$ is given by $\exp (s A), s \in \mathbb{R}$, with $A \in \mathfrak{g}$, the result follows easily.

Now, we focus our attention on the classification of the improper affine spheres which are invariant by a 1-parameter group of equiaffine transformations, that is, helicoidal improper affine spheres. For that, we need the following lemmas.

Lemma 5.1. Let $F_{s}, s \in \mathbb{R}$, be a 1-parameter group of equiaffine transformations in $\mathcal{G}$. Let us write, for each $s \in \mathbb{R}, F_{s}(v)=A(s) v+b(s)$, where $A(s)$ is a $3 \times 3$ matrix and $b(s) \in \mathbb{R}^{3}$. Let us also consider $\beta_{p}(s)=F_{s}(p)$ for $p \in \mathbb{R}^{3}$. Thus,

(1) Given $q_{1}=\left(q^{1}, q^{2}, q_{1}^{3}\right), q_{2}=\left(q^{1}, q^{2}, q_{2}^{3}\right) \in \mathbb{R}^{3}$, one has $\beta_{q_{1}}(s)=\beta_{q_{2}}(s)+$ $\left(0,0, q_{1}^{3}-q_{2}^{3}\right)$; that is, $\beta_{q_{1}}$ and $\beta_{q_{2}}$ agree up to a vertical translation. 
(2) If $p \in \mathbb{R}^{3}$ is such that $\beta_{p}$ is a regular analytic curve satisfying $\operatorname{det}\left(\beta_{p}^{\prime}, \beta_{p}^{\prime \prime}, e^{3}\right)$ $\neq 0$ everywhere, then for $m(s)=m_{0}>0$ the only improper affine sphere given by Proposition 3.1 is helicoidal.

(3) If $p \in \mathbb{R}^{3}$ and $V: \mathbb{R} \longrightarrow \mathbb{R}^{3}$ satisfy that $\left(\beta_{p}, V\right)$ is an admissible pair for $e^{3}$, then the improper affine sphere they generate is invariant under $F_{s}$ if and only if $V(s)=\left(A(s)^{t}\right)^{-1} V(0)$ for all $s \in \mathbb{R}$.

Proof. The first assertion is a direct computation. For the second statement, if we consider $F=F_{t}$ and $f(s)=s+t$ for an arbitrary constant $t \in \mathbb{R}$, then we have $F\left(\beta_{p}(s)\right)=\beta_{p}(f(s))$. Using Corollary 4.4, the improper affine sphere given by Proposition 3.1 remains unchanged by $F_{t}$.

Finally, for the third assertion, if $\psi$ is the improper affine sphere generated by $\beta_{p}$ and $V$ with affine conormal $N$, then we obtain that $F_{s} \circ \psi$ has affine conormal $\left(A(s)^{t}\right)^{-1} N$. Thus, since $\psi$ is invariant by the 1-parameter group and $F_{s}\left(\beta_{p}(0)\right)=$ $\beta_{p}(s)$, then $V(s)=\left(A(s)^{t}\right)^{-1} V(0)$. Conversely, from the generalized symmetry principle, it is sufficient to prove that for every $t \in \mathbb{R}$, the equiaffine transformation $F_{t}$ is a symmetry of the admissible pair. Thus, if we choose $f: \mathbb{R} \longrightarrow \mathbb{R}$ given by $f(s)=s+t$, then

$$
\begin{aligned}
& \beta_{p}(f(s))=F_{s+t}(p)=F_{t}\left(F_{s}(p)\right)=F_{t}\left(\beta_{p}(s)\right), \\
& V(f(s))=\left(A(s+t)^{t}\right)^{-1} V(0)=\left(A(t)^{t}\right)^{-1}\left(A(s)^{t}\right)^{-1} V(0)=\left(A(t)^{t}\right)^{-1} V(s),
\end{aligned}
$$

as we wanted to prove.

Lemma 5.2. Let $\psi: M \longrightarrow \mathbb{R}^{3}$ be an improper affine sphere with affine normal $e^{3}$, which is invariant under the 1-parameter group of equiaffine transformations $F_{s}, s \in \mathbb{R}$.

(1) Given a real number $\lambda$, the new immersion $\psi+\lambda e^{3}$ is also a helicoidal improper affine sphere invariant under $F_{s}$.

(2) Let $p \in \psi(M)$ and $\beta_{p}(s)=F_{s}(p)$. Then the affine metric $h$ satisfies that $h\left(\beta_{p}^{\prime}(s), \beta_{p}^{\prime}(s)\right)$ is constant.

Proof. Since $\psi$ has affine normal $e^{3}$, then $F_{s}$ is a 1-parameter subgroup of $\mathcal{G}$ and, so, the first statement follows easily. For the second assertion we observe that given an arbitrary fixed number $t \in \mathbb{R}, \beta_{p}(s+t)=F_{t}\left(\beta_{p}(s)\right)$ and then

$$
h\left(\beta_{p}^{\prime}(s+t), \beta_{p}^{\prime}(s+t)\right)=h\left(d F_{t}\left(\beta_{p}^{\prime}(s)\right), d F_{t}\left(\beta_{p}^{\prime}(s)\right)\right)=h\left(\beta_{p}^{\prime}(s), \beta_{p}^{\prime}(s)\right) .
$$

Here the second equality holds, because $h$ remains unchanged by equiaffine transformations.

From the above lemmas it seems clear that the classification of helicoidal improper affine spheres can be reached by studying the orbits of any point under the 1-parameter groups of equiaffine transformations. Thus, we focus our attention on the 1-parameter groups given by Proposition 5.1 .

\section{- Group (5.1):}

We distinguish two cases depending on the value of $b$ in the 1-parameter group (5.1).

If $b \neq 0$ and $p=\left(p^{1}, p^{2}, p^{3}\right) \in \mathbb{R}^{3}$, then the first coordinate immersion of the curve $\beta_{p}(s)$, given by the orbit of $p$, is $\beta_{p}^{1}(s)=p^{1}+b s$. Hence, every orbit has a point on the plane $\left\{\left(x^{1}, x^{2}, x^{3}\right): x^{1}=0\right\}$. 
On the other hand, by using Lemmas 5.1 and 5.2 the helicoidal improper affine spheres containing the point $\left(0, p^{2}, p^{3}\right)$ and the point $\left(0, p^{2}, 0\right)$ agree up to a vertical translation. Thus, the study of the helicoidal surfaces generated by the 1-parameter group (5.1) can be deduced from the study of the orbits of the points $\left(0, p^{2}, 0\right)$.

Let $p=(0, c, 0)$ and $\beta_{p}(s)=\left(b s, c+b s^{2} / 2, a c s+a b s^{3} / 6\right)$. If we take $m(s)=$ $m_{0}>0$, then from (3.6),

$$
V(s)=\left(-\frac{2 a c+2 m_{0} s-a b s^{2}}{2 b}, \frac{m_{0}-a b s}{b}, 1\right),
$$

and its associated improper affine sphere $\psi$ is (see Figure 1)

$$
\begin{aligned}
\psi^{1}(s, t) & =b s-a t \\
\psi^{2}(s, t) & =c+\frac{m_{0} t}{b}-a s t+\frac{b(s-t)(s+t)}{2}, \\
\psi^{3}(s, t)= & \frac{1}{6 b^{2}}\left(-2 t\left(3 m_{0}^{2}-3 b^{2} m_{0} t+b^{4} t^{2}\right)+a b s\left(6 b c+6 m_{0} t+b^{2}\left(s^{2}-3 t^{2}\right)\right)\right. \\
& \left.\quad-a^{2} b t\left(6 c+b\left(3 s^{2}+t^{2}\right)\right)\right) .
\end{aligned}
$$

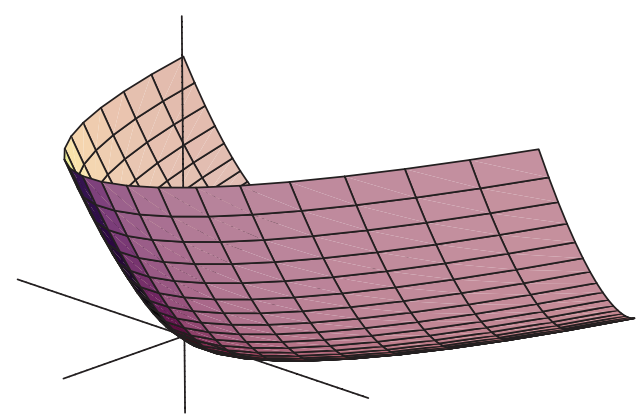

FiguRE 1. $a=0, b=1, c=0, m_{0}=1$.

Therefore, from Lemmas 5.1 and 5.2 , we obtain that every helicoidal improper affine sphere generated by the 1-parameter group (5.1), with $b \neq 0$, is given by (5.9), up to vertical translations.

Observe that its induced affine metric is given by

$$
h=\left(m_{0}-\left(a^{2}+b^{2}\right) t\right)\left(d s^{2}+d t^{2}\right) .
$$

Hence, $\psi$ is an immersion when $t<m_{0} /\left(a^{2}+b^{2}\right)$. Moreover, given a constant $r \in \mathbb{R}$, $\psi$ can be considered as a complete immersion in $\left\{(s, t): t \leq r<m_{0} /\left(a^{2}+b^{2}\right)\right\}$; that is, it is a complete immersion when we consider an orbit as its boundary.

Now, we assume $b=0$ in (5.1). Let $p=\left(0, p^{2}, p^{3}\right) \in \mathbb{R}^{3}$ and $\psi$ an improper affine sphere invariant by (5.1). If $p \in \psi(M)$, then there exists a point $q \in \psi(M)$ (in a neighbourhood of $p$ ) with nonzero first coordinate, and $\psi$ can be seen as an improper affine sphere generated by the orbit associated to $q$. Thus, the study of these helicoidal surfaces is reduced to the orbits of points with nonzero first coordinate. 
Let $p=\left(p^{1}, p^{2}, p^{3}\right) \in \mathbb{R}^{3}$ with $p^{1} \neq 0$. The second coordinate immersion of the curve $\beta_{p}(s)$, given by the orbit of $p$, is $\beta_{p}^{2}(s)=p^{1} s+p^{2}$. Hence, the orbit has a point on the plane $\left\{\left(x^{1}, x^{2}, x^{3}\right): x^{2}=0\right\}$.

By using again Lemmas 5.1 and 5.2 the helicoidal improper affine spheres containing the point $\left(p^{1}, 0, p^{3}\right)$ and the point $\left(p^{1}, 0,0\right)$ agree up to a vertical translation. Thus, the study of the helicoidal surfaces generated by the 1-parameter group (5.1), with $b=0$, can be deduced from the study of the orbits of the points $\left(p^{1}, 0,0\right)$, where $p^{1} \neq 0$.

Let $p=(c, 0,0), c \neq 0$ and $\beta_{p}(s)=\left(c, c s, a c s^{2} / 2\right)$. Let $V: \mathbb{R} \longrightarrow \mathbb{R}^{3}$ such that the pair $\beta_{p}, V$ is admissible for $e^{3}$ and generates a helicoidal improper affine sphere invariant by (5.1). Since $\operatorname{det}\left(\beta_{p}^{\prime}(s), \beta_{p}^{\prime \prime}(s), e^{3}\right) \equiv 0, V$ may be not unique, but using (3.1), $a c>0$ and $V(0)=(d, 0,1)$ with $d \in \mathbb{R}$, and using Lemma (5.1),

$$
V(s)=\left(d+\frac{a s^{2}}{2},-a s, 1\right) .
$$

In addition, the helicoidal improper affine sphere associated with the pair $\beta_{p}, V$ can be parametrized as (see Figure 2)

$$
\psi(s, t)=\left(c-a t, s(c-a t), \frac{a\left(6 d t+3 c\left(s^{2}+t^{2}\right)-a t\left(3 s^{2}+t^{2}\right)\right)}{6}\right)
$$

where $a c>0$. From Lemmas 5.1 and 5.2 every helicoidal improper sphere generated by the 1-parameter group (5.1), with $b=0$, is given by the above expression up to vertical translations.

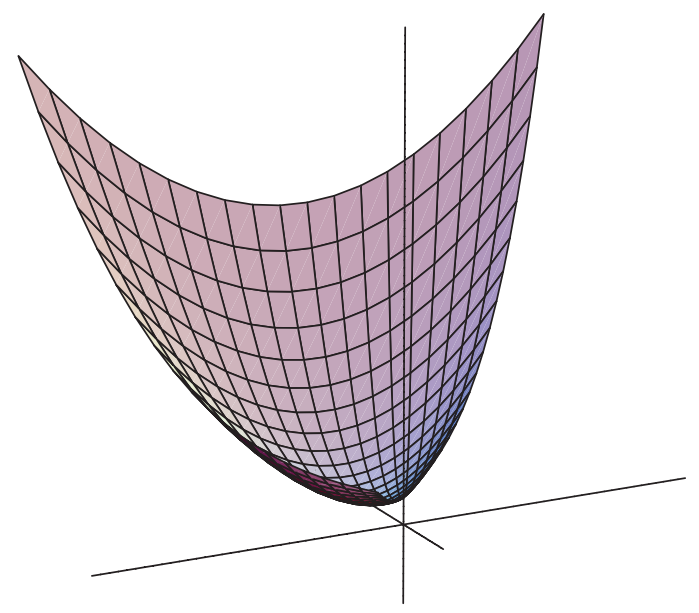

Figure 2. $a=1, c=1, d=0$.

Moreover, the induced affine metric of $\psi$ is given by

$$
h=a(c-a t)\left(d s^{2}+d t^{2}\right),
$$

so that $\psi$ is an immersion when $t<c / a$. Thus, $\psi$ is a complete immersion in $\{(s, t): t \leq r<c / a\}$ where $r \in \mathbb{R}$; that is, it is a complete immersion when we consider an orbit as its boundary.

The study of the rest of the 1-parameter groups can be achieved in a similar way. Hence, we will omit some redundant comments in the following cases. 


\section{- Groups (5.2), (5.7) and (5.8):}

There is no improper affine sphere generated by one of these 1-parameter groups since almost every orbit is a straight line and an improper affine sphere must be locally strongly convex.

\section{- Group (5.3):}

The study of the helicoidal improper affine spheres associated with (5.3) can be reduced to the orbits of the points $\left(p^{1}, p^{2}, p^{3}\right)$ with $\left(p^{1}\right)^{2} \neq\left(p^{2}\right)^{2}$. Up to a rotation of $k \pi / 2$ with $k=1,2,3$, and using Lemmas 5.1 and 5.2 we only need to investigate the orbits of the points $p=(c, 0,0)$, where $c>0$.

Thus, one has $\beta_{p}(s)=(c \cosh s, c \sinh s, a s)$ and, given $m(s)=m_{0}>0$, we obtain $V(s)=\left(\left(m_{0} \cosh s+a \sinh s\right) / c,-\left(a \cosh s+m_{0} \sinh s\right) / c, 1\right)$. The helicoidal improper affine sphere that they generate can be parametrized by (see Figure 3 )

$$
\begin{aligned}
& \psi^{1}(s, t)=c \cos t \cosh s-\frac{\sin t\left(m_{0} \cosh s+a \sinh s\right)}{c}, \\
& \psi^{2}(s, t)=c \cos t \sinh s-\frac{\sin t\left(a \cosh s+m_{0} \sinh s\right)}{c} \\
& \psi^{3}(s, t)=\frac{1}{4}\left(2 m_{0}+4 a s+\frac{2\left(-a^{2}+c^{4}+m_{0}^{2}\right) t}{c^{2}}\right. \\
&\left.-2 m_{0} \cos (2 t)-\frac{\left(a^{2}+c^{4}-m_{0}^{2}\right) \sin (2 t)}{c^{2}}\right) .
\end{aligned}
$$

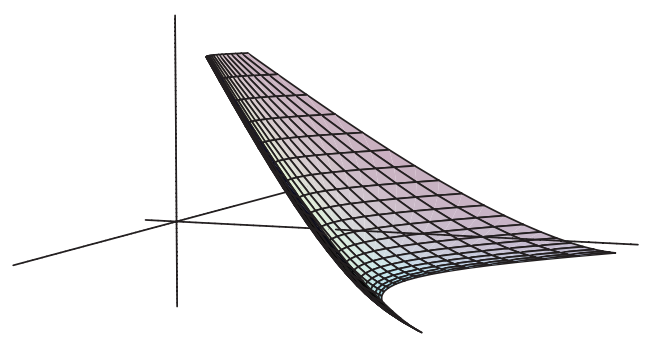

FiguRE 3. $a=0, c=1, m_{0}=1$.

Its affine metric

$$
h=\left(m_{0} \cos (2 t)+\left(a^{2}+c^{4}-m_{0}^{2}\right) \cos t \sin t / c^{2}\right)\left(d s^{2}+d t^{2}\right)
$$

is not complete if we consider an orbit as its boundary.

\section{- Group (5.4):}

The study of the helicoidal improper affine spheres associated with (5.4) can be reduced to the orbits of the points $p=(c, 0,0)$ with $c>0$. Thus, one obtains $\beta_{p}(s)=(c \cos s, c \sin s, a s)$, and given $m(s)=m_{0}>0$, then

$$
V(s)=\left(\left(-m_{0} \cos s+a \sin s\right) / c,-\left(a \cos s+m_{0} \sin s\right) / c, 1\right) .
$$


The associated helicoidal improper affine sphere that they generate can be parametrized by (see Figure 4)

$$
\begin{gathered}
\psi^{1}(s, t)=c \cos s \cosh t+\frac{\left(-m_{0} \cos s+a \sin s\right) \sinh t}{c}, \\
\psi^{2}(s, t)=c \cosh t \sin s-\frac{\left(a \cos s+m_{0} \sin s\right) \sinh t}{c} \\
\psi^{3}(s, t)=\frac{1}{4}\left(-2 m_{0}+4 a s-\frac{2\left(a^{2}-c^{4}+m_{0}^{2}\right) t}{c^{2}}\right. \\
\left.+2 m_{0} \cosh (2 t)-\frac{\left(a^{2}+c^{4}+m_{0}^{2}\right) \sinh (2 t)}{c^{2}}\right) .
\end{gathered}
$$

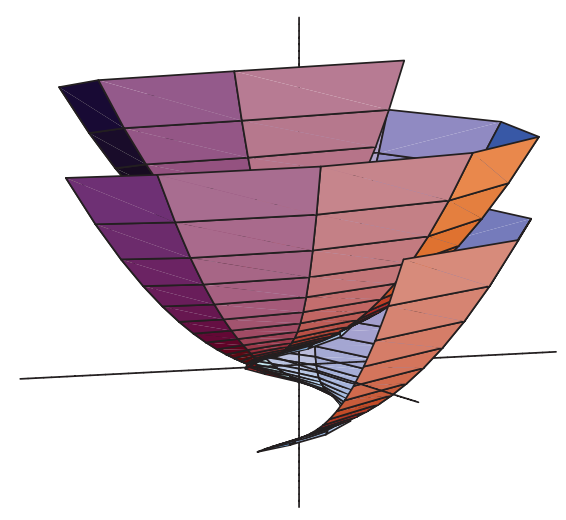

Figure 4. $a=1, c=1, m_{0}=1$.

Its affine metric is

$$
h=\left(m_{0} \cosh (2 t)-\left(\left(a^{2}+c^{4}+m_{0}^{2}\right) \cosh t \sinh t\right) / c^{2}\right)\left(d s^{2}+d t^{2}\right)
$$

and, so, $\psi$ is well defined for $t<\operatorname{arctanh}\left(2 c^{2} m_{0} /\left(a^{2}+c^{4}+m_{0}^{2}\right)\right) / 2$. Thereby, $\psi$ can be seen as a complete immersion with an orbit as its boundary.

We observe that $h$ is well defined for all $(s, t) \in \mathbb{R}^{2}$ if and only if $a=0$ and $m_{0}=c^{2}$. Or equivalently, the immersion can be parametrized as $\left(x^{1}, x^{2}, f\left(x^{1}, x^{2}\right)\right)$, where $f\left(x^{1}, x^{2}\right)=\left(\left(x^{1}\right)^{2}+\left(x^{2}\right)^{2}\right) / 2-c^{2} / 2$; that is, $\psi$ is an elliptic paraboloid.

\section{- Group (5.5):}

The study of the helicoidal improper affine spheres associated to (5.5) can be reduced to the orbits of the points $p=(c, c, 0)$ with $c>0$. Thus, one obtains $\beta_{p}(s)=\left(c e^{s}, c e^{-s}, a s\right)$, and, given $m(s)=m_{0}>0$, then

$$
V(s)=\left(\left(m_{0}-a\right) e^{-s} /(2 c),\left(m_{0}+a\right) e^{s} /(2 c), 1\right)
$$


The associated helicoidal improper affine sphere that they generate can be parametrized by (see Figure 5)

$$
\begin{aligned}
\psi^{1}(s, t)= & \frac{e^{s}\left(2 c^{2} \cos t+\left(a+m_{0}\right) \sin t\right)}{2 c}, \\
\psi^{2}(s, t)= & \frac{e^{-s}\left(2 c^{2} \cos t+\left(-a+m_{0}\right) \sin t\right)}{2 c}, \\
\psi^{3}(s, t)= & \frac{4 c^{2}\left(m_{0}+2 a s\right)+2\left(a^{2}-4 c^{4}-m_{0}^{2}\right) t}{8 c^{2}} \\
& \frac{-4 c^{2} m_{0} \cos (2 t)+\left(a^{2}+4 c^{4}-m_{0}^{2}\right) \sin (2 t)}{8 c^{2}} .
\end{aligned}
$$

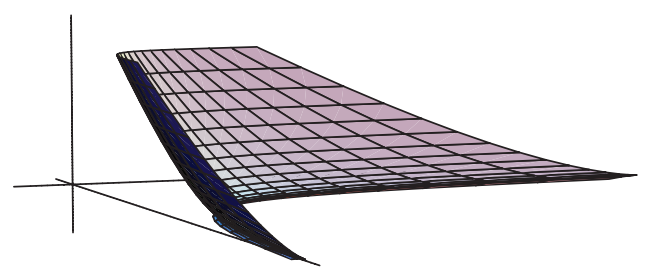

FiguRE 5. $a=0, c=1, m_{0}=1$.

Its affine metric is

$$
h=\left(m_{0} \cos (2 t)-\left(\left(a^{2}+4 c^{4}-m_{0}^{2}\right) \sin (2 t)\right) /\left(4 c^{2}\right)\right)\left(d s^{2}+d t^{2}\right)
$$

and, so, $\psi$ is not complete if we consider an orbit as its boundary.

\section{- Group (5.6):}

The helicoidal improper affine spheres associated to (5.6) can be investigated from the orbits of the points $p=(0, c, 0)$ with $c \in \mathbb{R}$. Thus, one gets $\beta_{p}(s)=$ $\left(s, c, b c s+a s^{2} / 2\right)$, and $V(s)$ must be $(-b c-a s, d-b s, 1)$ for an arbitrary $d \in \mathbb{R}$ with $a>0$, from Lemma 5.1 .

The associated helicoidal improper affine sphere that they generate can be parametrized by

$$
\psi(s, t)=\left(s-b t, c+a t, \frac{1}{2}\left(2 b c s-2 b^{2} c t+a\left(s^{2}-2 d t+t^{2}\right)\right)\right)
$$

that is, the immersion can be reparametrized as $\left(x^{1}, x^{2}, f\left(x^{1}, x^{2}\right)\right)$, where $f\left(x^{1}, x^{2}\right)$ is a polynomial of second order. Hence, the improper affine sphere $\psi$ is an elliptic paraboloid.

Finally, we summarize the study of helicoidal improper spheres in the following result.

Theorem 5.1. Let $\psi$ be a helicoidal improper affine sphere. Then, up to equiaffine transformations, $\psi$ is given by (5.9), (5.10), (5.11), (5.12) or (5.13).

In addition, if $\psi$ can be considered as a complete immersion with an orbit as its boundary, then $\psi$ can only be isometric to (5.9), (5.10) or (5.12). 


\section{Classification of the nOnRemovable Singularities}

We devote this section to the study of the singularities of the improper affine spheres and the Hessian one equation. For that, we will need a slightly more general conformal representation for the class of improper affine spheres with singularities.

First, we recall the relationship between special Lagrangian immersions and improper affine spheres in order to obtain an adequate singularity concept.

Let us denote by $\mathbb{C}^{2} \equiv \mathbb{R}^{4}$ the complex 2-plane and let $\left(x^{1}, x^{2}, x^{3}, x^{4}\right)$ be a rectangular coordinate system in $\mathbb{R}^{4}$. Taking $y^{1}=x^{1}+i x^{2}, y^{2}=x^{3}+i x^{4}$, we consider the usual metric $g$, the symplectic form $\omega$ and the complex 2 -form $\Omega$ given by

$$
g=\left|d y^{1}\right|^{2}+\left|d y^{2}\right|^{2}, \quad \omega=\frac{i}{2}\left(d y^{1} \wedge d \overline{y^{1}}+d y^{2} \wedge d \overline{y^{2}}\right), \quad \Omega=d y^{1} \wedge d y^{2} .
$$

Then, the following facts were proved in [Ma (see also $[\mathrm{Wo}$ ).

- If $M$ is a Riemann surface and $\psi=\left(\psi^{1}, \psi^{2}, \psi^{3}\right): M \longrightarrow \mathbb{R}^{3}$ is an improper affine sphere with affine normal $e^{3}$ and affine conormal $N=\left(N^{1}, N^{2}, 1\right)$, then the map $L^{\psi}: M \longrightarrow \mathbb{C}^{2}$ given by $\left(\psi^{1}+i \psi^{2}, N^{1}+i N^{2}\right)$ is a special Lagrangian immersion with respect to the calibration $\Re(i \Omega)$. Moreover, its induced metric $d \tau^{2}=d\left(\psi^{1}\right)^{2}+d\left(\psi^{2}\right)^{2}+d\left(N^{1}\right)^{2}+d\left(N^{2}\right)^{2}$ is conformal to the affine metric $h$ of $\psi$.

- Conversely, if $L: M \longrightarrow \mathbb{C}^{2}$ is a special Lagrangian immersion with respect to the calibration $\Re(i \Omega)$ given by $L(z)=\left(y^{1}(z), y^{2}(z)\right), z \in M$, then

$$
\psi^{L}(z)=\left(\Re\left(y^{1}(z)\right), \Im\left(y^{1}(z)\right),-\int_{z_{0}}^{z}\left\langle d y^{1}, y^{2}\right\rangle\right)
$$

is a (possibly multivalued) improper affine sphere at its regular points, where $z_{0}$ is a fixed point. In addition, the immersion is locally a graph $\left(x^{1}, x^{2}, f\left(x^{1}, x^{2}\right)\right)$ satisfying (2.1). If we define

$$
N^{L}(z)=\left(\Re\left(y^{2}(z)\right), \Im\left(y^{2}(z)\right), 1\right) \quad \text { and } \quad h^{L}=\left\langle d \psi^{L},-d N^{L}\right\rangle,
$$

then they are the affine conormal and the affine metric when $f_{x^{1} x^{1}}>0$ and they change signs when $f_{x^{1} x^{1}}<0$.

Roughly speaking, the above map $\psi^{L}$ is an improper affine sphere with "natural" singularities. Concretely, $\psi^{L}$ has a singularity at a point $p$ if and only if the affine metric is degenerate and $d \tau^{2}$ is regular at $p$.

Observe that given an improper affine sphere $\psi$ with affine normal $e^{3}$ and associated special Lagrangian immersion $L^{\psi}$, then the new immersion $\psi^{L^{\psi}}$ agrees with $\psi$, up to a vertical translation.

In fact, a map $\psi: M \longrightarrow \mathbb{R}^{3}$ is called an improper affine map or an improper affine sphere with admissible singularities if there exists a special Lagrangian immersion $L: M \longrightarrow \mathbb{C}^{2}$ with respect to the calibration $\Re(i \Omega)$ such that its associated map $\psi^{L}$ agrees with $\psi$, up to a vertical translation.

Obviously, the map $\psi^{L}$ gives us (possibly multivalued) solutions to the MongeAmpère equation (2.1) with singularities. In particular, we will focus our attention on the classification of the nonremovable isolated singularities of the Hessian one equation. 
In the spirit of Theorem 3.1 we obtain the following result for special Lagrangian immersions:

Theorem 6.1. Let $I$ be an interval and $\gamma: I \longrightarrow \mathbb{R}^{4} \equiv \mathbb{C}^{2}$ a regular analytic curve:

$$
\gamma(s)=\left(\beta^{1}(s), \beta^{2}(s), V^{1}(s), V^{2}(s)\right) \in \mathbb{R}^{4}, \quad s \in I .
$$

Then there exists a unique special Lagrangian immersion $L$ with respect to the calibration $\Re(i \Omega)$ containing $\gamma(I)$. Moreover, $L=\left(L^{1}, L^{2}, L^{3}, L^{4}\right)$ can be calculated in a neighbourhood of $I$ in $\mathbb{C}$ as

$$
\begin{aligned}
& L^{1}(z)=\Re\left(\beta^{1}(z)-i V^{2}(z)\right), \\
& L^{2}(z)=\Im\left(-V^{1}(z)+i \beta^{2}(z)\right), \\
& L^{3}(z)=-\Re\left(-V^{1}(z)+i \beta^{2}(z)\right), \\
& L^{4}(z)=-\Im\left(\beta^{1}(z)-i V^{2}(z)\right),
\end{aligned}
$$

with

$$
d \tau^{2}=\left(\left|\beta_{z}^{1}-i V_{z}^{2}\right|^{2}+\left|V_{z}^{1}-i \beta_{z}^{2}\right|^{2}\right)|d z|^{2}
$$

its induced metric.

In addition, the associated improper affine sphere with admissible singularities $\psi^{L}$ is given by

$$
\psi^{L}(z)=\left(L^{1}(z), L^{2}(z),-\int_{s_{0}}^{z}\left(L_{w}^{1} L^{3}+L_{w}^{2} L^{4}\right) d w+\left(L_{\bar{w}}^{1} L^{3}+L_{\bar{w}}^{2} L^{4}\right) d \bar{w}\right),
$$

where $s_{0} \in I$ is a fixed point, and the affine metric at its regular points is

$$
h=\left|\Re\left(\left(\beta_{z}^{1}-i V_{z}^{2}\right)\left(\overline{V_{z}^{1}}+i \overline{\beta_{z}^{2}}\right)\right)\right||d z|^{2} .
$$

Proof. Bearing in mind that the functions $L^{1}-i L^{4}$ and $-L^{3}+i L^{2}$ are holomorphic, the proof of this result is, in essence, the one given in Theorem 3.1 and Theorem 3.2 .

Remark 6.1. As was observed in Remark 3.1, the special Lagrangian immersion $L$ and the improper affine sphere with admissible singularities $\psi^{L}$ can be recovered by analytic continuation not only on a neighbourhood of $I$ but globally.

As an interesting consequence of the above result we obtain that, under some assumptions, an immersion can be recovered in terms of its set of singularities:

Corollary 6.1. Let $I$ be an interval and $\beta: I \longrightarrow \mathbb{R}^{3}$ a regular analytic curve satisfying $\operatorname{det}\left(\beta^{\prime}(s), \beta^{\prime \prime}(s), e^{3}\right) \neq 0$, for all $s \in I$. Then, there exists a unique improper affine map containing $\beta(I)$ in its set of singularities.

Proof. From Theorem 6.1, if $\beta=\left(\beta^{1}, \beta^{2}, \beta^{3}\right)$ is a curve of singularities, then there exists a Lagrangian immersion $L$ and a local conformal parameter $z=s+i t$ such that

$$
L(s)=\left(\beta^{1}(s), \beta^{2}(s), V^{1}(s), V^{2}(s)\right), \quad s \in I,
$$

for certain analytic functions $V^{1}, V^{2}$, and $\psi^{L}(s)=\beta(s)$, up to a vertical translation.

Therefore, from (6.3),

$$
-\beta^{3^{\prime}}(s)=\beta^{1^{\prime}}(s) V^{1}(s)+\beta^{2^{\prime}}(s) V^{2}(s) .
$$

Now, the affine metric is degenerate on $\beta$ if and only if

$$
0=\beta^{1^{\prime}}(s) V^{1^{\prime}}(s)+\beta^{2^{\prime}}(s) V^{2^{\prime}}(s) .
$$


Besides, by differentiation in (6.5), the above equation is equivalent to

$$
-\beta^{3^{\prime \prime}}(s)=\beta^{1^{\prime \prime}}(s) V^{1}(s)+\beta^{2^{\prime \prime}}(s) V^{2}(s) .
$$

Hence, from (6.5), (6.6) and since $\operatorname{det}\left(\beta^{\prime}(s), \beta^{\prime \prime}(s), e^{3}\right) \neq 0$ for all $s \in I$, one has that $V^{1}$ and $V^{2}$ are uniquely determined. Thus, $L(s)$ is known and the uniqueness and existence of the improper affine map is clear from Theorem 6.1.

Example 6.1. Let us consider the regular analytic curve given by

$$
\beta(s)=\left(\cos s, \sin s, \frac{1}{5} \cos (2 s)\right), \quad s \in \mathbb{R} .
$$

Then, since $\operatorname{det}\left(\beta^{\prime}(s), \beta^{\prime \prime}(s), e^{3}\right) \equiv 1$, there exists a unique improper affine map containing $\beta(\mathbb{R})$ in its set of singularities, which can be calculated by solving (6.5), (6.6), and taking

$$
L(s)=\left(\cos s, \sin s,-\frac{4}{5} \cos ^{3} s, \frac{4}{5} \sin ^{3} s\right)
$$

in Theorem 6.1

This surface can be parametrized by (see Figure 6)

$$
\begin{aligned}
& \psi^{1}= \cos s \cosh t+\frac{3 \cos s \sinh t}{5}-\frac{\cos (3 s) \sinh (3 t)}{5}, \\
& \psi^{2}= \cosh t \sin s-\frac{3 \sin s \sinh t}{5}-\frac{\sin (3 s) \sinh (3 t)}{5}, \\
& \psi^{3}=\frac{1}{100}(62 t+10 \cos (2 s)(3-2 \cosh (2 t)+\cosh (4 t)) \\
&\left.\quad-24 \cos (4 s) \cosh ^{3} t \sinh t-16 \sinh (2 t)-\sinh (6 t)\right) .
\end{aligned}
$$

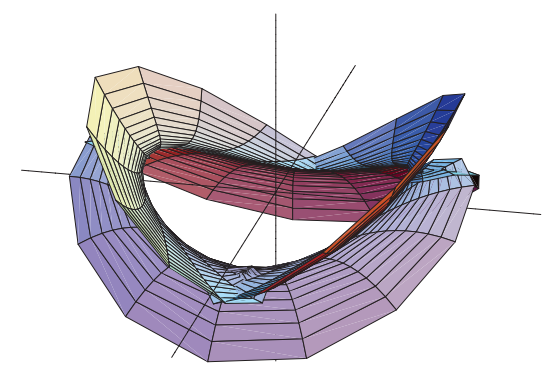

FIGURE 6. Improper affine sphere with admissible singularities.

Finally, we focus our attention on the classification of the isolated singularities of the Monge-Ampère equation (2.1). The classification of the entire solutions to the Hessian one equation with a finite number of isolated singularities is achieved in GMMi]. Nevertheless, nothing is known about the local classification of these singularities. Thus, as the main application of Theorem 6.1, we give an explicit description of the solutions to (2.1) around an isolated singularity.

Let $\mathcal{U}$ be a neighbourhood of $(0,0)$ in the $\left(x^{1}, x^{2}\right)$-plane and $f\left(x^{1}, x^{2}\right)$ a $\mathcal{C}^{2}$ solution (and so analytic solution) to (2.1) on $\mathcal{U} \backslash\{(0,0)\}$. Then $f$ extends continuously to $(0,0)$; moreover, if this extension is $\mathcal{C}^{1}$ at $(0,0)$, then $f$ is globally $\mathcal{C}^{2}$ on $\mathcal{U}$ (see 
GMMi] and [Jo2 ). Therefore, it is said that $f$ has a nonremovable singularity at $(0,0)$ if $f$ is not $\mathcal{C}^{1}$ at this point.

Proposition 6.1. Let $\mathcal{U}$ be a neighbourhood of $(0,0)$ in the $\left(x^{1}, x^{2}\right)$-plane and $f: \mathcal{U} \longrightarrow \mathbb{R} a \mathcal{C}^{2}$ solution to (2.1) on $\mathcal{U} \backslash\{(0,0)\}$, with $f(0,0)=0$. Then there exists a $2 \pi$-periodic regular analytic curve $V: \mathbb{R} \longrightarrow \mathbb{R}^{2}$ such that the graph $\left(x^{1}, x^{2}, f\left(x^{1}, x^{2}\right)\right)$ can be parametrized around the origin by

$$
\psi(z)=\left(L^{1}(z), L^{2}(z),-\int_{0}^{z}\left(L_{w}^{1} L^{3}+L_{w}^{2} L^{4}\right) d w+\left(L_{\bar{w}}^{1} L^{3}+L_{\bar{w}}^{2} L^{4}\right) d \bar{w}\right)
$$

for $z \in B_{r_{0}}=\left\{w \in \mathbb{C}: 0 \leq \Im(w)<r_{0}\right\}$, where

$L^{1}(z)=\Im\left(V^{2}(z)\right), L^{2}(z)=-\Im\left(V^{1}(z)\right), L^{3}(z)=\Re\left(V^{1}(z)\right), L^{4}(z)=\Re\left(V^{2}(z)\right)$

with $V=\left(V^{1}, V^{2}\right)$ and $r_{0} \in \mathbb{R}$.

Here, $\psi$ is an immersion when $0<\Im(z)<r_{0}$ and $\psi(z)=(0,0,0)$ when $\Im(z)=0$. Moreover, $\psi$ is well defined on the annulus $B_{r_{0}} /(2 \pi \mathbb{Z})$, i.e., $\psi(z+2 \pi)=\psi(z)$ for any $z \in B_{r_{0}}$.

Proof. It is proved in [Jo2] (see also GMMi]) that $f$ has a nonremovable singularity at $(0,0)$ if and only if the underlying conformal structure of its affine metric around the singularity is that of an annulus $A_{r}=\{w \in \mathbb{C}: 1<|w|<r\}$.

Thus, if we consider the special Lagrangian immersion $L$ associated to the improper affine sphere $\left(x^{1}, x^{2}, f\left(x^{1}, x^{2}\right)\right)$ in this punctured neighbourhood of $(0,0)$, then $L$ is not only well defined on the annulus $A_{r}$ but it can be analytically continued across $|w|=1$ by Schwarzian reflection on $\{w \in \mathbb{C}: 1 / r<|w|<r\}$ (see GMMi]). In addition, if we denote by $\psi^{L}=\left(\psi^{1}, \psi^{2}, \psi^{3}\right)$ the improper affine map associated to $L$, then $\left(\psi^{1}, \psi^{2}\right)(w)=(0,0)$ for $|w|=1$ (i.e., the singularity is the image of $|w|=1)$.

Let us consider the new conformal parametrization $\widetilde{L}(z)=L\left(e^{-i z}\right)$ with $-\log r$ $<\Im(z)<\log r$. Then if we write $z=s+i t$ and

$$
\widetilde{L}(s)=\left(\beta^{1}(s), \beta^{2}(s), V^{1}(s), V^{2}(s)\right), \quad s \in \mathbb{R},
$$

it is clear that $\widetilde{L}(s+2 \pi)=\widetilde{L}(s)$ and $\left(\beta^{1}(s), \beta^{2}(s)\right)=\left(\psi^{1}\left(e^{-i s}\right), \psi^{2}\left(e^{-i s}\right)\right)=(0,0)$.

Hence, for $0 \leq \Im(z)<\log r$, the improper affine map $\psi^{\widetilde{L}}$ is a $2 \pi$-periodic parametrization of $\left(x^{1}, x^{2}, f\left(x^{1}, x^{2}\right)\right)$ around the origin, in such a way that $\psi^{\widetilde{L}}(s)=$ $(0,0,0)$ for all $s \in \mathbb{R}$. Here $\psi^{\widetilde{L}}$ is recovered from Theorem 6.1 for $\widetilde{L}(s)=\left(0,0, V^{1}(s)\right.$, $\left.V^{2}(s)\right)$ as we wanted to prove.

Thus, the local study of nonremovable singularities of (2.1) is reduced to the investigation of the properties of the closed curve $V$.

First, we observe that given a $2 \pi$-periodic regular analytic curve

$$
\gamma(s)=\left(0,0, V^{1}(s), V^{2}(s)\right), \quad s \in \mathbb{R},
$$

then the special Lagrangian immersion that $\gamma$ generates, $L: \Delta \longrightarrow \mathbb{R}^{4} \equiv \mathbb{C}^{2}$, satisfies $L(z+2 \pi)=L(z)$, for $z, z+2 \pi \in \Delta$, and it is easy to see that the same is true for its associated improper affine map $\psi^{L}: \Delta \longrightarrow \mathbb{R}^{3}$, where $\Delta$ stands for a neighbourhood of $\mathbb{R}$ in $\mathbb{C}$.

From the periodicity of the data we can assume the existence of a real number $r_{0}$ such that $\Delta_{0}:=\left\{z \in \mathbb{C}:-r_{0}<\Im(z)<r_{0}\right\} \subseteq \Delta$. In addition, since $V^{i}$ is real 
analytic, $V^{i}(\bar{z})=\overline{V^{i}(z)}$ for $i=1,2, z \in \Delta_{0}$. Therefore,

$$
L(\bar{z})=\left(L_{1}(\bar{z}), L_{2}(\bar{z}), L_{3}(\bar{z}), L_{4}(\bar{z})\right)=\left(-L_{1}(z),-L_{2}(z), L_{3}(z), L_{4}(z)\right), \quad z \in \Delta_{0},
$$

and

$$
\psi^{L}(\bar{z})=-\psi^{L}(z)
$$

That is, if $\psi^{L}$ parametrizes the solution $\left(x^{1}, x^{2}, f\left(x^{1}, x^{2}\right)\right)$ for $0 \leq \Im(z)<r_{0}$ (as in Proposition 6.1), then $\psi^{L}$ is a parametrization of the solution $\left(y^{1}, y^{2}, g\left(y^{1}, y^{2}\right)\right)=$ $-\left(x^{1}, x^{2}, f\left(x^{1}, x^{2}\right)\right)$ for $-r_{0}<\Im(z) \leq 0$.

Subsequently, we remark that to consider a new analytic parameter $\widetilde{s}=\widetilde{s}(s)$ for the curve $\gamma$ is nothing but a conformal change of parameter for $L$ from $z=s+i t$ to $\widetilde{z}=\widetilde{s}+i \widetilde{t}$ and hence a different parametrization of $\psi^{L}$.

Nevertheless, it is important to bear in mind that if $\widetilde{s}^{\prime}(s)>0$, then the points with $\Im(z)>0$ correspond to those with $\Im(\widetilde{z})>0$, but if $\widetilde{s}^{\prime}(s)<0$, then the points with $\Im(z)>0$ correspond to those with $\Im(\widetilde{z})<0$. That is, if $V(s)$ is a $2 \pi$ periodic parametrization associated to $\left(x^{1}, x^{2}, f\left(x^{1}, x^{2}\right)\right)$ and we consider the new $2 \pi$-periodic parametrization $V(\widetilde{s})$, then we obtain a new $2 \pi$-periodic parametrization of $\left(x^{1}, x^{2}, f\left(x^{1}, x^{2}\right)\right)$ using Proposition 6.1 if the new parameter preserves the orientation of the plane curve $V\left(\widetilde{s}^{\prime}(s)>0\right)$. Otherwise, we obtain a parametrization of the reflected solution $-\left(x^{1}, x^{2}, f\left(x^{1}, x^{2}\right)\right)$.

With all of this, we note that the solution $\left(x^{1}, x^{2}, f\left(x^{1}, x^{2}\right)\right)$ only depends on the curve $V(\mathbb{R})$, where we identify the solution with its reflected one.

Thus, if we consider $\mathcal{S}$ as the set of local solutions $f\left(x^{1}, x^{2}\right)$ to (2.1) with nonremovable singularity around the origin such that $f(0,0)=0$, where a solution and its reflected one are identified, one has

Theorem 6.2 (Classification of the nonremovable singularities). There exists an explicit one-to-one correspondence between $\mathcal{S}$ and the (strongly) convex regular analytic Jordan curves of $\mathbb{R}^{2}$.

Proof. First of all, we need to prove that given a solution $f\left(x^{1}, x^{2}\right)$ to (2.1) around the origin with nonremovable singularity at $(0,0)$ and $f(0,0)=0$, then the $2 \pi$ periodic regular analytic plane curve $V: \mathbb{R} \longrightarrow \mathbb{R}^{2}$ given by Proposition 6.1 has curvature different from zero at any point, or equivalently, $V(\mathbb{R})=V([0,2 \pi[)$ is a locally (strongly) convex closed curve. In addition we will show that it is a Jordan curve. This correspondence is clearly injective since $V$ determines $f$ and later we will show that it is also surjective.

Let $V=\left(V^{1}, V^{2}\right): \mathbb{R} \longrightarrow \mathbb{R}^{2}$ be a $2 \pi$-periodic regular analytic curve. Associated to $\gamma(s)=\left(0,0, V^{1}(s), V^{2}(s)\right)$ we consider the special Lagrangian immersion $L$ which is well defined on $\Delta_{0}:=\left\{z=s+i t \in \mathbb{C}:-r_{0}<\Im(z)<r_{0}\right\}$ for a certain $r_{0} \in \mathbb{R}$.

From the expression of the affine metric of $\psi^{L}$ (6.4) one has that every real number is a nonregular point. In fact, $z \in \Delta_{0}$ is nonregular if and only if

$$
0=\Re\left(-i V_{z}^{2} \overline{V_{z}^{1}}\right)=\frac{1}{4}\left(\left|V_{z}^{2}+i V_{z}^{1}\right|^{2}-\left|V_{z}^{2}-i V_{z}^{1}\right|^{2}\right) .
$$

Thus, the set of singular points of $\psi^{L}$ is the nodal set of the harmonic function

$$
\omega=\log \left(\frac{\left|V_{z}^{2}+i V_{z}^{1}\right|^{2}}{\left|V_{z}^{2}-i V_{z}^{1}\right|^{2}}\right) .
$$

That is, since the real line $\mathbb{R}$ is made up of singular points, it follows that, close enough to $\mathbb{R}, \psi^{L}$ is an immersion if and only if $\mathbb{R}$ is not crossed by any other nodal curve of $\omega$, or equivalently, $\omega_{z}(s) \neq 0$ for all $s \in \mathbb{R} \subseteq \mathbb{C}$. 
As

$$
\omega_{z}=2 i \frac{V_{z z}^{1} V_{z}^{2}-V_{z}^{1} V_{z z}^{2}}{\left(V_{z}^{1}\right)^{2}+\left(V_{z}^{2}\right)^{2}}
$$

then for $s_{0} \in \mathbb{R}$ one has that $\omega_{z}\left(s_{0}\right) \neq 0$ if and only if $V$ is locally convex at $s_{0}$.

Therefore, given a solution $f\left(x^{1}, x^{2}\right)$ to (2.1) around the origin with a nonremovable singularity at $(0,0)$ and $f(0,0)=0$, then its associated plane curve $V$ given by Proposition 6.1 must be locally convex.

We need to show that $V$ is, in fact, globally convex, and so we will have a welldefined correspondence from $\mathcal{S}$ into the set of convex regular analytic Jordan curves of $\mathbb{R}^{2}$. For that, we will use the Legendre transform of an immersion (see [LSZ, p. 89]).

Let $\psi=\left(\psi^{1}, \psi^{2}, \psi^{3}\right): B_{r_{0}} /(2 \pi \mathbb{Z}) \longrightarrow \mathbb{R}^{3}$ be the parametrization of the graph of $f\left(x^{1}, x^{2}\right)$ given by Proposition 6.1. Since this graph is identified with its reflected solution, we can assume that $f_{x^{1} x^{1}}>0$. Thus, from (6.1) and (6.2), the affine conormal of $\psi$ at its regular points is $\left(N^{1}, N^{2}, N^{3}\right)=\left(\Re\left(V^{1}(z)\right), \Re\left(V^{2}(z)\right), 1\right)$ and the Legendre transform of $\psi$ is given by

$$
\mathcal{T}^{\psi}=-\left(N^{1}, N^{2}, \psi^{1} N^{1}+\psi^{2} N^{2}+\psi^{3}\right) .
$$

Observe that $\mathcal{T}^{\psi}$ is well defined on the annulus $B_{r_{0}} /(2 \pi \mathbb{Z})$ and is a locally strongly convex immersion for $0<\Im(z)<r_{0}$ because so is $\psi$. In addition, its affine conormal is given by

$$
\mathcal{N}^{\mathcal{T}}=\left(-\psi^{1},-\psi^{2}, 1\right) .
$$

Let us denote by $C_{r}$ the circle $\{z \in \mathbb{C}: \Im(z)=r\} /(2 \pi \mathbb{Z})$. The third coordinate $\mathcal{T}^{3}$ of $\mathcal{T}^{\psi}$ extends continuously to $C_{0}$, so $\mathcal{T}^{3}\left(C_{0}\right)=0$, and the affine conormal also extends continuously to the circle $C_{0}$ with $\mathcal{N}^{\mathcal{T}}\left(C_{0}\right)=(0,0,1)$.

Then, since $\mathcal{T}^{\psi}$ is locally strongly convex, there exists an annulus $\mathcal{A}=\{z \in$ $\left.\mathbb{C}: 0<\Im(z) \leq r_{1}\right\} /(2 \pi \mathbb{Z})$, with $r_{1}<r_{0}$, satisfying that $\mathcal{T}^{\psi}(\mathcal{A})$ is properly immersed in the upper half-space $\mathbb{R}_{+}^{3}=\left\{\left(x^{1}, x^{2}, x^{3}\right): x^{3}>0\right\}$. In particular, if $d_{0}=\operatorname{dist}\left(0, \mathcal{T}^{3}\left(C_{r_{1}}\right)\right)$, then for all $d \in\left(0, d_{0}\right)$ the curves $\Gamma_{d}=\mathcal{T}^{\psi}(\mathcal{A}) \cap\left\{\left(x^{1}, x^{2}, x^{3}\right)\right.$ : $\left.x^{3}=d\right\}$ are regular, convex and closed.

Now, we prove that these curves $\Gamma_{d}$ are embedded. If the planar curve $\Gamma_{d}$ was not embedded, then the Euclidean normal vector $n_{\Gamma_{d}}$ of $\Gamma_{d}$ on this plane would wind at least twice. But the orthogonal projection of the conormal along $\Gamma_{d}$ on $\left\{\left(x^{1}, x^{2}, x^{3}\right): x^{3}=d\right\}$ has the same direction as $n_{\Gamma_{d}}$. So, the affine conormal along $\Gamma_{d}$ would wind at least twice on the plane $\left\{\left(x^{1}, x^{2}, x^{3}\right): x^{3}=1\right\}$. This would mean that $\mathcal{N}^{\mathcal{T}}$ self-intersects along $\Gamma_{d}$. But, this is not possible, since by (6.9) it would contradict that $\left(x^{1}, x^{2}, f\left(x^{1}, x^{2}\right)\right)$ is a graph because of the existence of two different points with the same $\left(x^{1}, x^{2}\right)$ coordinates.

Therefore, all the curves $\Gamma_{d}=\left(\Gamma_{d}^{1}, \Gamma_{d}^{2}, d\right)$ are regular convex Jordan curves lying on parallel planes. Thus, we obtain a family of regular convex Jordan curves $V_{d}=$ $\left(\Gamma_{d}^{1}, \Gamma_{d}^{2}, 0\right)$ which converges to $\mathcal{T}^{\psi}\left(C_{0}\right)=\left(V^{1}, V^{2}, 0\right)$ when $d \rightarrow 0$. Hence, $V$ is an embedded curve as we wanted to show.

To complete the proof, we only need to prove that the correspondence is surjective.

Let $V=\left(V^{1}, V^{2}\right): \mathbb{R} \longrightarrow \mathbb{R}^{2}$ be a $2 \pi$-periodic regular analytic parametrization of a convex Jordan curve and $L$ the special Lagrangian immersion generated by $\gamma(s)=\left(0,0, V^{1}(s), V^{2}(s)\right)$, which is well defined if $-r_{0}<\Im(z)<r_{0}$ for a certain real number $r_{0}$. 
Using the above study of the nodal set of $V$, the $2 \pi$-periodic improper affine map $\psi^{L}$ is an immersion when $0<\Im(z)<r_{1}$ for a suitable $r_{1}<r_{0}$. That is, $\psi(w)=\psi^{L}(i \log w)$ is a well-defined immersion on $\mathcal{A}^{\prime}=\left\{w \in \mathbb{C}: 1<|w|<e^{r_{1}}\right\}$ with $\psi(w)=(0,0,0)$ for $|w|=1$. Moreover, if $\psi$ can be seen as a graph for $1 \leq|w|<e^{r_{1}}$, then $(0,0)$ is a nonremovable singularity since its induced conformal structure is the one of an annulus.

We can assume that the affine normal of $\psi$ on $\mathcal{A}^{\prime}$ is $e^{3}$, up to an identification with its reflected solution. Thus, the Legendre transform $\mathcal{T}^{\psi}$ of $\psi$, given by (6.8), takes the limit values $\left(V^{1}, V^{2}, 0\right)$ for $|w|=1$, with constant limit affine conormal $(0,0,1)$.

Therefore, $\mathcal{T}^{\psi}$ lies in $\mathbb{R}_{+}^{3}$, and there is some $d_{0}>0$ such that for every $d \in$ $\left(0, d_{0}\right]$ the intersection $\Gamma_{d}=\mathcal{T}^{\psi}\left(\mathcal{A}^{\prime}\right) \cap\left\{\left(x^{1}, x^{2}, x^{3}\right): x^{3}=d\right\}$ is a regular convex Jordan curve. Now, let $S_{d_{1}}$ denote the portion of $\mathcal{T}^{\psi}\left(\mathcal{A}^{\prime}\right)$ which lies in the slab $\left\{\left(x^{1}, x^{2}, x^{3}\right): d_{1} \leq x^{3} \leq d_{0}\right\}$, where $d_{1}>0$. Then, as $S_{d_{1}}$ is convex, it is known that the convexity and embeddedness of the curves $\Gamma_{d}$ assure that the Euclidean unit normal on $S_{d_{1}}$ is a global diffeomorphism onto its spherical image. Letting $d_{1} \rightarrow 0$, the Euclidean normal of $\mathcal{T}^{\psi}$ is a global diffeomorphism from $1<|w|<r_{2}$ onto its spherical image, for a certain $r_{2}$. In particular, the affine conormal of $\mathcal{T}^{\psi}$ is a global diffeomorphism from $1<|w|<r_{2}$.

Hence, by (6.9) we conclude that $\psi$ must be a global graph about the origin, as we wished to prove.

\section{REFERENCES}

[ACM] L.J. Alías, R.M.B. Chaves and P. Mira, Björling problem for maximal surfaces in the Lorentz-Minkowski space, Math. Proc. Cambridge 134 (2003), 289-316. MR1972140 (2004d:53076)

[Bl1] W. Blaschke, Über affine Geometrie. XXXIII: Affinminimalflächen, Math. Z. 12 (1922), 262-273. MR 1544517

[B12] W. Blaschke, Vorlesungen über Differentialgeometrie, II, Berlin, Springer, 1923.

[Ca] E. Calabi, Hypersurfaces with maximal affinely invariant area, Amer. J. Math. 104 (1984), 91-126. MR0648482 (85b:53054)

[CY] S.Y. Cheng and S.T. Yau, Complete affine hypersurfaces (part I), Comm. Pure Appl. Math. 39 (1986), 839-866. MR0859275 (87k:53127)

[DHKW] U. Dierkes, S. Hildebrandt, A. Küster and O. Wohlrab, Minimal Surfaces I. A series of comprehensive studies in mathematics, Springer Verlag, 295, Berlin, 1992. MR.1215267 (94c:49001a)

[FMM1] L. Ferrer, A. Martínez and F. Milán, Symmetry and Uniqueness of Parabolic Affine Spheres, Math. Ann. 305 (1996), 311-327. MR.1391217 (97c:53018)

[FMM2] L. Ferrer, A. Martínez and F. Milán, An extension of a theorem by K. Jörgens and a maximum principle at infinity for parabolic affine spheres, Math. Z. 230 (1999), 471-486. MR:1679973 (2001d:53010)

[GMM] J.A. Gálvez, A. Martínez and F. Milán, Flat surfaces in the hyperbolic 3-space, Math. Ann. 316 (2000), 419-435. MR1752778 (2002b:53013)

[GMMi] J.A. Gálvez, A. Martínez and P. Mira, The space of solutions to the Hessian one equation in the finitely punctured plane, J. Math. Pure Appl. 84 (2005), 1744-1757. MR.2180389 (2006g:35064)

[GMi1] J.A. Gálvez and P. Mira, Dense solutions to the Cauchy problem for minimal surfaces, B. Braz. Math. Soc. 35 (2004), 387-394. MR2106312(2005j:53010)

[GMi2] J.A. Gálvez and P. Mira, The Cauchy problem for the Liouville equation and Bryant surfaces, Adv. Math. 195 (2005), 456-490. MR2146351 (2006i:53003)

[GMi3] J.A. Gálvez and P. Mira, Embedded isolated singularities of flat surfaces in hyperbolic 3-space, Calc. Variations \& PDEs 24 (2005), 239-260. MR2164928 (2006i:53014) 
[Jo1] K. Jörgens, Über die Lösungen der Differentialgleichung $r t-s^{2}=1$, Math. Ann. 127 (1954), 130-134. MR0062326 (15:961e)

[Jo2] K. Jörgens, Harmonische Abbildungen und die Differentialgleichung $r t-s^{2}=1$, Math. Ann. 129 (1955), 330-344. MR0073823 (17:493b)

[LSZ] A.M. Li, U. Simon and G. Zhao, Global Affine Differential Geometry of Hypersurfaces, Walter de Gruyter, Berlin, 1993. MR1257186 (95e:53016)

[Ma] A. Martínez, Improper Affine Maps, Math. Z. 249 (2005), 755-766. MR2126213 (2006a:53010)

[Mi] P. Mira, Complete minimal Möbius strips in $\mathbb{R}^{n}$ and the Björling problem, J. Geom. Phys. 56 (2006), 1506-1515. MR2240407 (2007d:53012)

[Ni] J.C.C. Nitsche, Lectures on Minimal Surfaces, Vol. I. Cambridge University Press, Cambridge, 1989. MR:1015936 (90m:49031)

[NS] K. Nomizu and T. Sasaki, Affine Differential Geometry, Cambridge Univ. Press, Cambridge, 1994. MR.1311248 (96e:53014)

[Sp] M. Spivak, A comprehensive introduction to Differential Geometry, Vol. 4, second edition, Publish or Perish, Inc., Wilmington, DE, 1979. MR.0532833 (82g:53003d)

[Wo] J. Wolfson, Minimal Lagrangian diffeomorphisms and the Monge-Ampère equation, $J$. Differential Geom. 46 (1997), 335-373. MR1484047|(99e:58045)

Departamento de Matemáticas, Universidad de Castilla-La Mancha, 02071 Albacete, SPAIN

E-mail address: juanangel.aledo@uclm.es

Instituto de Matemática e Estatística, Universidade de São Paulo, 05315-970 São PAUlO-SP, BRAZIL

E-mail address: rosab@ime.usp.br

Departamento de Geometría y Topología, Universidad de Granada, 18071 Granada, SPAIN

E-mail address: jagalvez@ugr.es 